\title{
Identification of New Potential APE1 Inhibitors by Pharmacophore Modeling and Molecular Docking
}

\author{
In Won Lee ${ }^{1}$, Jonghwan Yoon ${ }^{2}$, Gunhee Lee ${ }^{2}$, Minho Lee $^{3 *}$ \\ ${ }^{1}$ Department of Biological Science, Sangji University, Wonju 26339, Korea, ${ }^{2}$ Department of Biomedicine \& Health Sciences, \\ Graduate School, The Catholic University of Korea, Seoul 06591, Korea, ${ }^{3}$ Catholic Precision Medicine Research Center, \\ College of Medicine, The Catholic University of Korea, Seoul 06591, Korea
}

\begin{abstract}
Apurinic/apyrimidinic endonuclease 1 (APE1) is an enzyme responsible for the initial step in the base excision repair pathway and is known to be a potential drug target for treating cancers, because its expression is associated with resistance to DNA-damaging anticancer agents. Although several inhibitors already have been identified, the identification of novel kinds of potential inhibitors of APE1 could provide a seed for the development of improved anticancer drugs. For this purpose, we first classified known inhibitors of APE1. According to the classification, we constructed two distinct pharmacophore models. We screened more than 3 million lead-like compounds using the pharmacophores. Hits that fulfilled the features of the pharmacophore models were identified. In addition to the pharmacophore screen, we carried out molecular docking to prioritize hits. Based on these processes, we ultimately identified 1,338 potential inhibitors of APE1 with predicted binding affinities to the enzyme.
\end{abstract}

Keywords: APE1, molecular docking simulation, pharmacophore modeling, virtual screening

\section{Introduction}

DNA damage occurs naturally and due to the environment, altering the cell's abilities that are encoded by the DNA, and may lead to diseases, like cancer. Cells respond to DNA damage by DNA repair and cellular apoptosis $[1,2]$. Apurinic/apyrimidinic endonuclease (APE) is an enzyme that identifies damaged apurinic/apyrimidinic sites in DNA, cuts the phosphodiester bond in the backbone of the sites, and has critical roles in the base excision pathway [3]. APE1 has recently been noted as a potential drug target for treating cancer, in that overexpression of the enzyme has been observed and shown to be associated with a poor response to cancer treatment, such as radiation and anticancer drugs, and a lower overall survival rate [4-7]. Antineoplastic agents that are to treat cancers are known to induce the expression of APE1, increasing the resistance of tumor cells to drug treatment. Thus, compounds that inhibit the activity of APE1 could be potential anticancer drugs with DNA-damaging antineoplastic agents used in the clinic [8].
For this reason, there have been several attempts to develop compounds targeting APE1. Currently, although there is no approved drug yet, three candidates - 7-nitroindole-2-carboxylic acid (also known as CRT0044876) [9, 10], lucanthone (also known as Miracil D) [9], and methoxyamine (trademark TRC102)-are known to inhibit APE1 activity and are under examination in clinical trials. Lucanthone and CRT0044876 have rings similar to the deoxyribose sugar ring without a base and many hydrogen bond acceptors that can interact with hydrogen bond donors in the active site of APE1. These properties lead APE1 to stick in the site and prevent it from repairing DNA damage [11]. Methoxyamine is known to attack the open-ring form of AP sites to form an oxime linkage. In other words, methoxyamine blocks APE1 from accessing the lesion site rather than targeting the enzyme directly. This may lead to nonspecific off-target effects $[12,13]$. Although several inhibitors of APE1 have been discovered, most potent compounds have weaknesses [14]. Thus, it is necessary to find novel kinds of potential inhibitors targeting APE1. Here, we present out work, in which we applied pharmacophore modeling and 


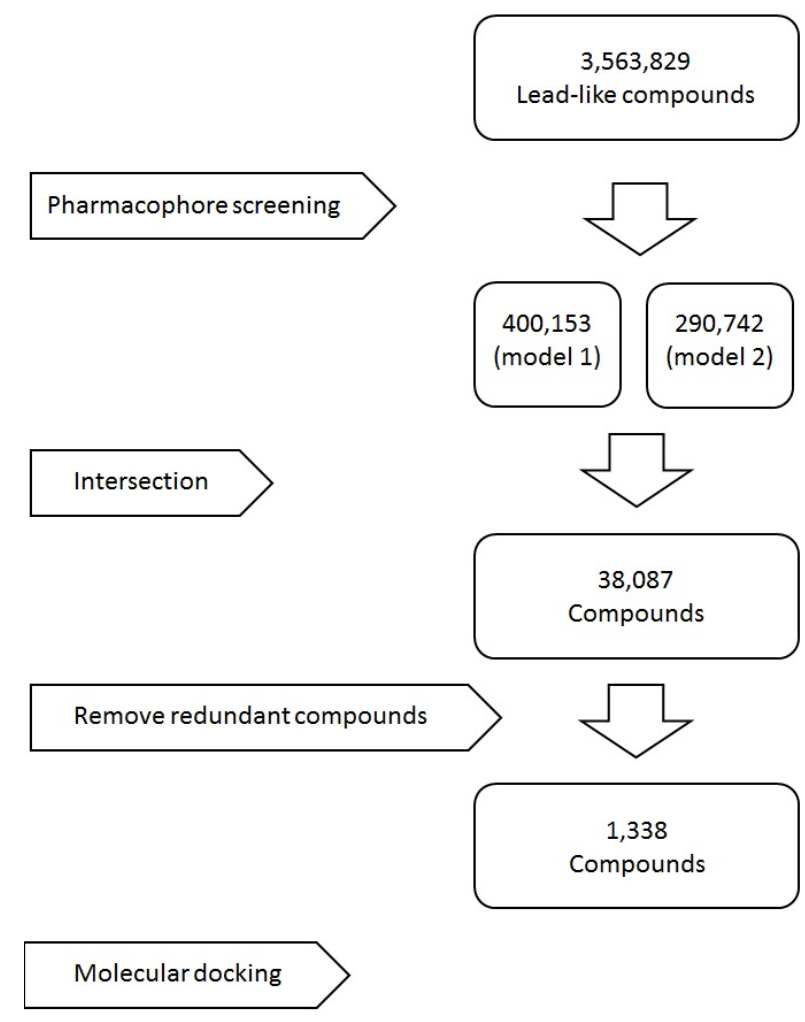

Fig. 1. Outline of overall procedures of the screen to find potential inhibitors of apurinic/apyrimidinic endonuclease 1 . virtual screening. The overall procedures we carried out are illustrated in Fig. 1. We constructed pharmacophore models by capturing the common features of known inhibitors of APE1. The modes were used to screen a vast number of lead-like compounds, and molecular docking was used to prioritize the hits of the screen.

\section{Methods}

\section{Selection of ligands for pharmacophore modeling}

From the ChEMBL [15] database, we retrieved 52 compounds known to be targets of APE1 and 51 compounds with an $\mathrm{IC}_{50}$ of less than $10 \mu \mathrm{M}$. By eliminating redundancy, the number of compounds was reduced into 83. The list did not contain methoxyamine; so, methoxyamine was also added to the list.

We clustered these 84 compounds by Tanimoto distance, based on the PubChem fingerprint [16], and finally categorized them into two groups by excluding two outliers (CHEMBL1213633 and CHEMBL313493) (Fig. 2). A total of 49 molecules in group 1 (Fig. 3) and 33 molecules in group 2 (Fig. 4) were used to generate pharmacophore models 1 and 2 , respectively.

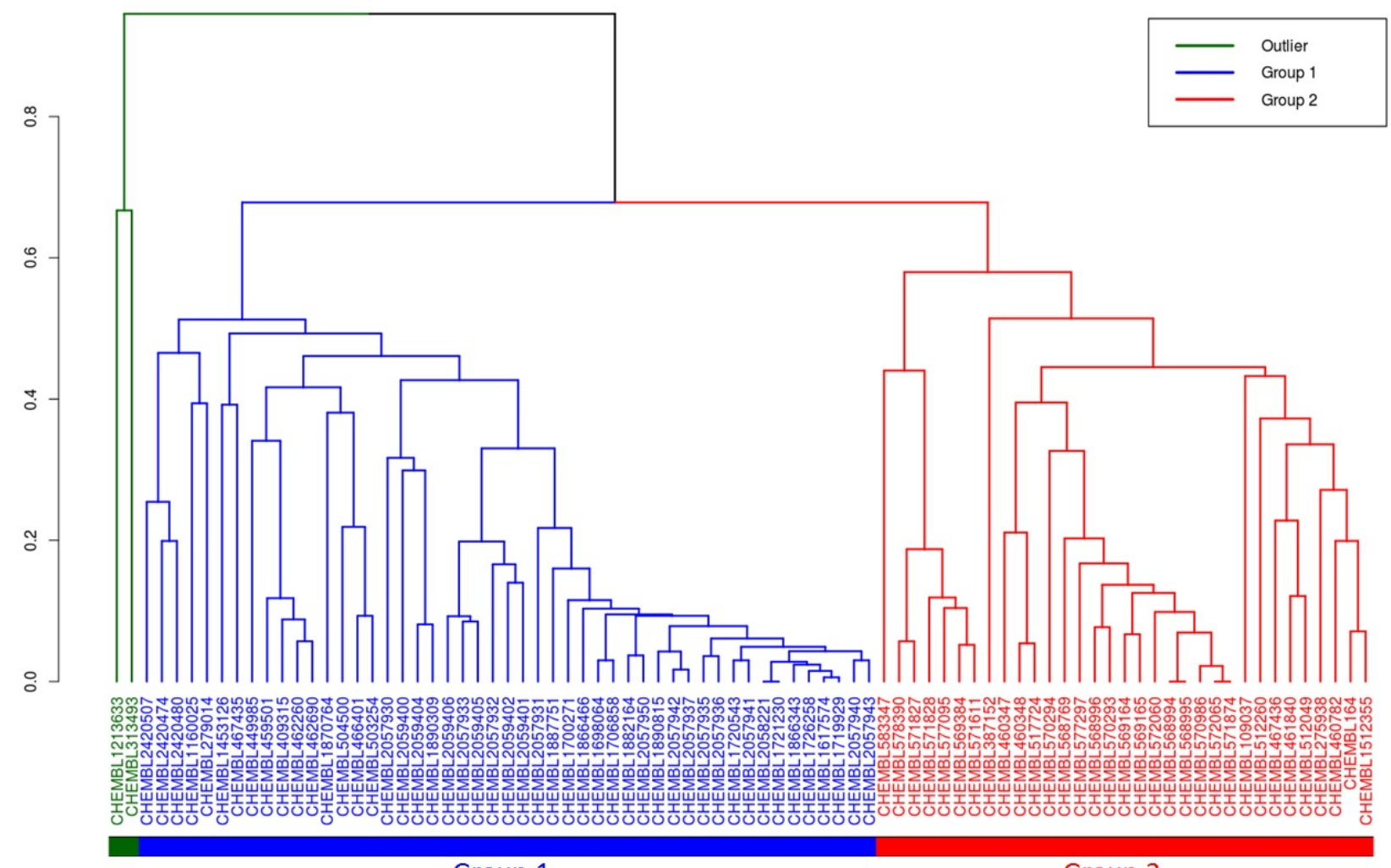

Group 1

Group 2

Fig. 2. Clustering of molecules for construction of pharmacophore models. 


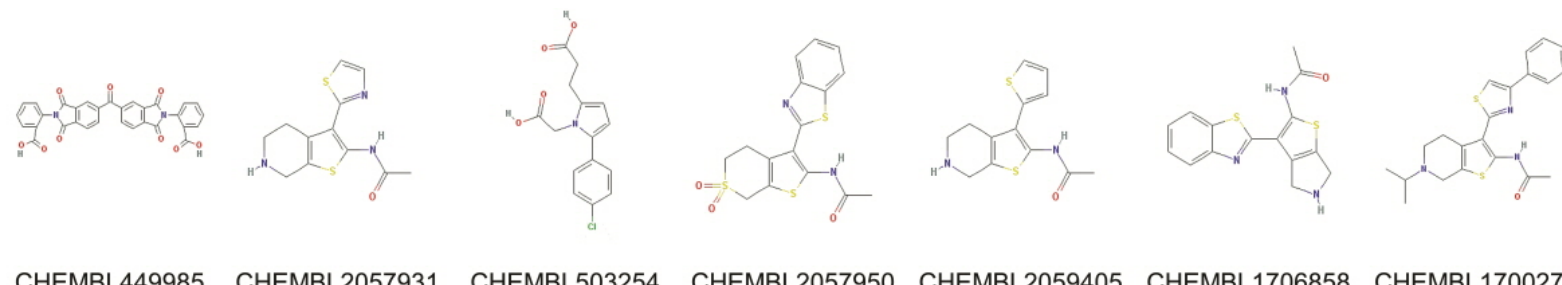

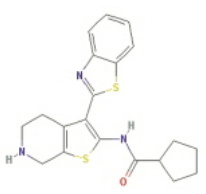

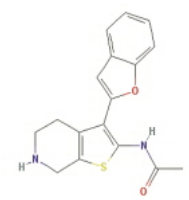

CHEMBL2057936

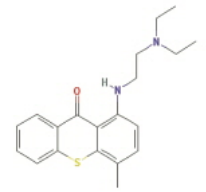

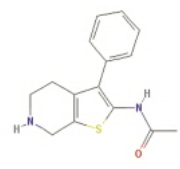

CHEMBL279014<smiles>CC(C)C1CCC(c2ccccc2)C(C(C)Cc2ccccc2)C1C(C)Cc1ccccc1</smiles>

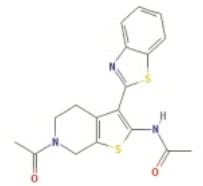

CHEMBL2057937

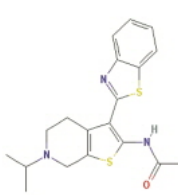

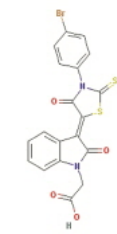

CHEMBL1617574

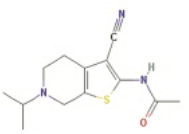

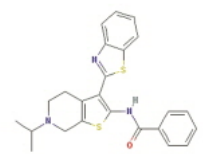

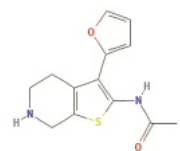

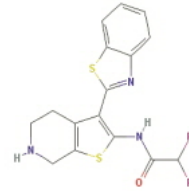

CHEMBL2057932 CHEMBL1890815

CHEMBL2059404

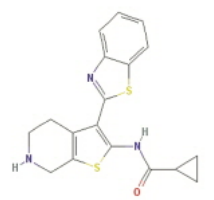<smiles>CC(C)C1CCCC2CCCCC21</smiles>

CHEMBL1870764

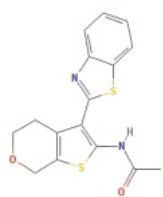

CHEMBL2420507 CHEMBL1887751

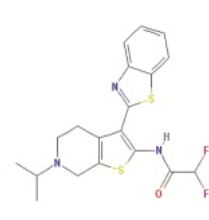

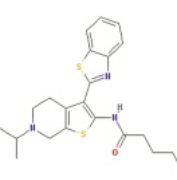

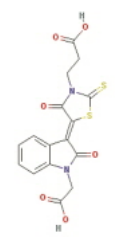

CHEMBL462690

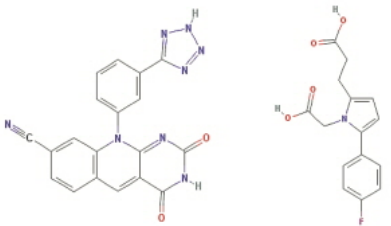

CHEMBL2420480 CHEMBL466401

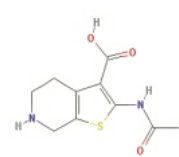

CHEMBL2059400

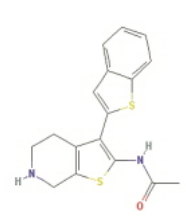

CHEMBL2059406<smiles></smiles>

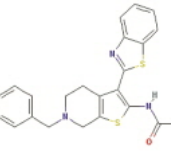

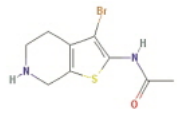

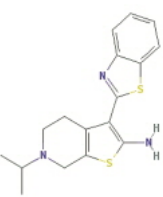

CHEMBL1866466
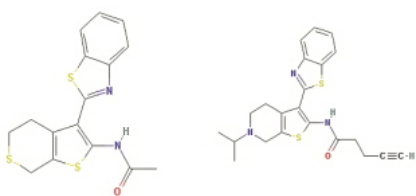

CHEMBL504500

CHEMBL1882164 CHEMBL2057940
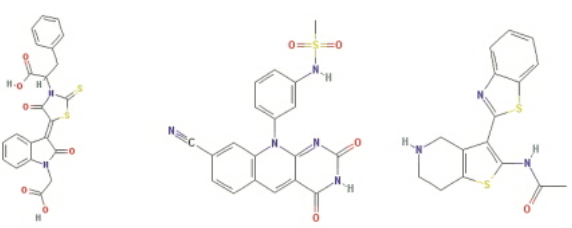

CHEMBL409315

CHEMBL2420474 CHEMBL2058221

Fig. 3. Structures of the 49 compounds in group 1 used to generate pharmacophore model 1. 

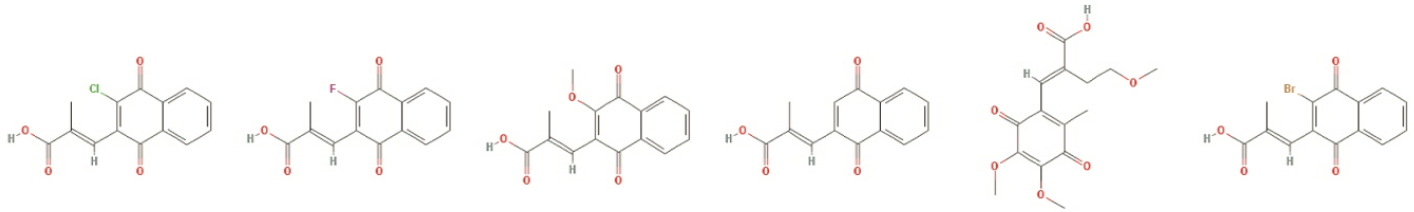

CHEMBL577095

CHEMBL568996

CHEMBL571828
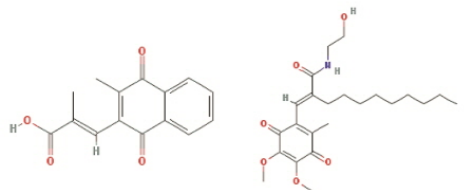

CHEMBL571874

CHEMBL583347

CHEMBL460348

CHEMBL568769

CHEMBL570294

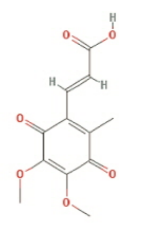

CHEMBL569384

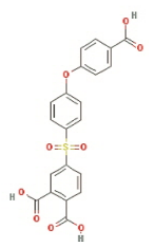

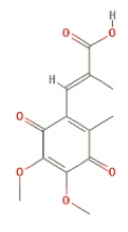

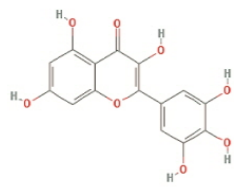

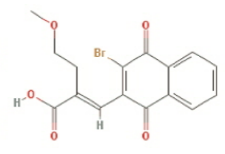

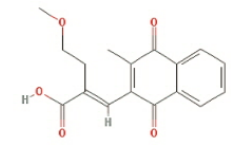

CHEMBL568995

CHEMBL569165

CHEMBL460347

CHEMBL571611

CHEMBL164

CHEMBL570293

CHEMBL569164<smiles>CCCCCCC(C(C)C)C(C)C(C)C(C)C(C)C</smiles>

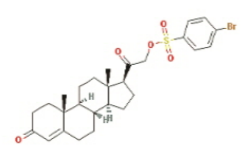

CHEMBL571827 CHEMBL387152

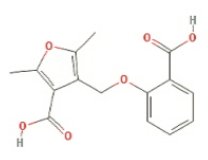

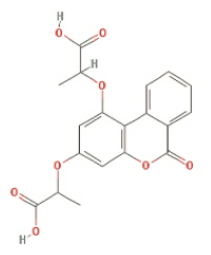

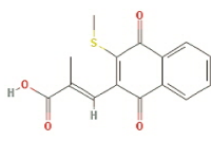

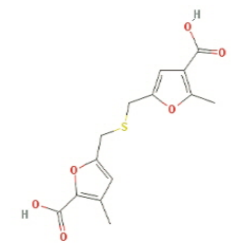

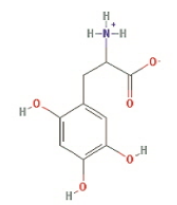

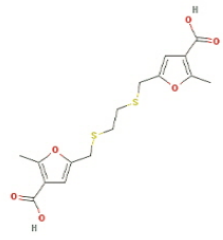

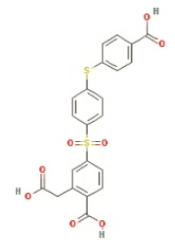

CHEMBL461840

Fig. 4. Structures of the 33 compounds in group 2 used to generate pharmacophore model 2 .

\section{Generation of pharmacophore model}

Ligandscout tools 4.1 [17] was used to generate the ligand-based pharmacophore models. Ligandscout is known to be able to increase the selectivity of a pharmacophore model with the excluded volume feature. To generate more flexible pharmacophore, the threshold of the portion of partially matching features was set to $20 \%$.

\section{Pharmacophore screen}

For the initial set of the pharmacophore screen, we selected a lead-like subset [18], defined by the ZINC database (ZINC is not commercial) [19]. Similar to druglikeness [20], like Lipinski's rule of 5 [21], lead-like compounds are defined as being large enough to be validated in experiments but are smaller than most drugs, optimized too specifically, and more soluble than their drug-like compounds. ZINC provides a lead-like subset fulfilling leadlikeness as follows: (1) molecular weight between 250 and $350 \mathrm{Da}$, 


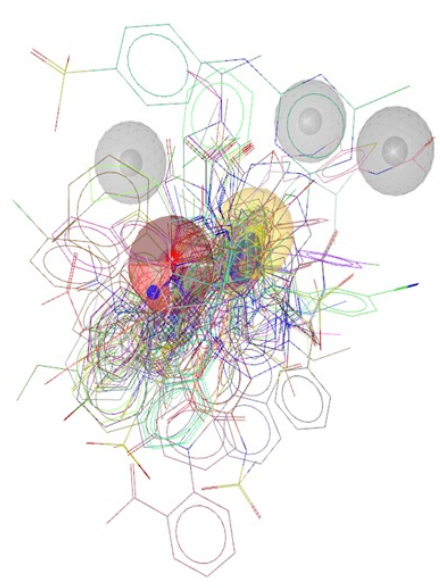

(A)

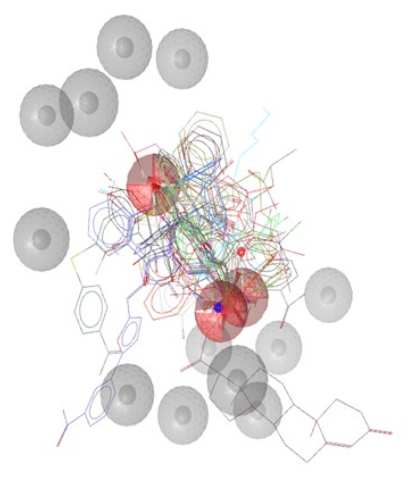

(B)
Pharmacophore features

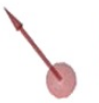

Hydrogen bond acceptor

Hydrophobic interactions

\section{Excluded volume}

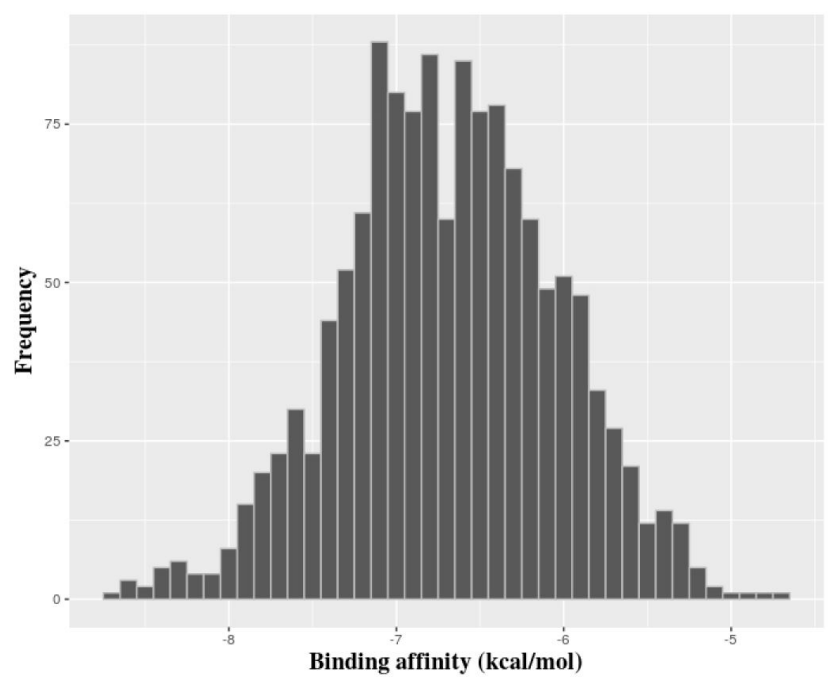

Fig. 6. Distribution of predicted binding affinities by molecular docking of potential inhibitors to apurinic/apyrimidinic endonuclease 1.

(2) partition coefficient $\log \mathrm{p} \leq 3.5$, and (3) no more than seven rotatable bonds. The structures of lead-like compounds in medium $\mathrm{pH}$ were downloaded and converted into a database for screening by idbgen, a component of Ligand-

\section{Aromatic ring}

Negative ionizable area

Fig. 5. Pharmacophore models used for screening. Models were generated by compounds in group 1 (A) and group 2 (B).

scout. We carried out pharmacophore screens using iscreen in Ligandscout for models 1 and 2 independently. Pharmacophore fit scores were also calculated by LigandScout based on the number of matching pharmacophore features and the root-mean-square deviation of the pharmacophore alignment.

\section{Molecule docking simulation}

To prioritize the hits of the pharmacophore screen, we docked the hits against previously determined structures of APE1 [22] (PDB ID: 1DEW) using AutoDock Vina [23]. A binding site of APE1 was assigned using the fpocket algorithm [24].

\section{Results and Discussion}

A total of 84 compounds from the ChEMBL database were first collected to generate a pharmacophore, but their structures and properties were too heterogeneous to get common features. Thus, we carried out clustering and categorized the compounds into two groups (Figs. 2-4). For each group of compounds, we generated the corresponding pharmacophore model. Pharmacophore model 1 was generated by 49 compounds from group 1 . The model was 
IW Lee, et al. Identification of Novel APE1 Inhibitors

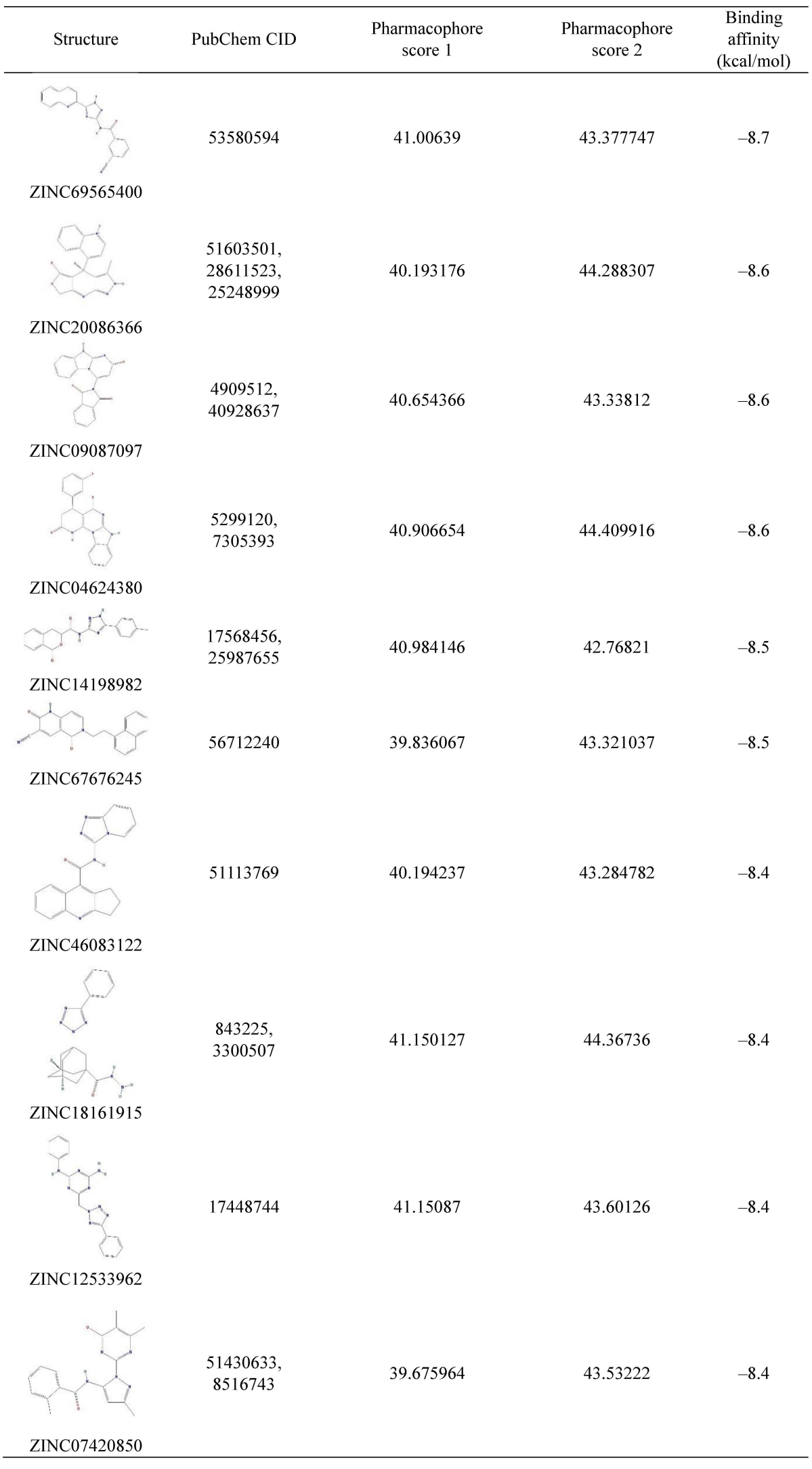

Fig. 7. Structures and scores of the screen of the top 10 hits. 
composed of four features (one hydrophobic centroid, one aromatic ring, two hydrogen acceptors) and three exclusion volume spaces (Fig. 5A). Model 2 was generated by 33 compounds from group 2 . The model was composed of four features (one negative ionizable and three hydrogen bond acceptors) and 12 exclusion volume spaces (Fig. 5B).

For 3,563,829 lead-like compounds retrieved from the ZINC database, we performed a pharmacophore screen based on pharmacophore models 1 and 2 independently. Among multiple subsets provided by ZINC, we chose the lead-like subset, not the drug-like set, because we aimed to provide a list of potential hits that could be optimized further by other groups, as well as our group.

As a result, 400,153 and 290,742 hits fulfilled the features of models 1 and 2, respectively. The intersection of the two lists of hits, which fulfilled all features of both models, consisted of 38,087 compounds. To remove structurally similar compounds, we clustered the 38,087 hits by hierarchical clustering, based on the Tanimoto distance in PubChem Fingerprint. According to the result of the clustering, we ruled out redundant compounds that had similar compounds (Tanimoto coefficient >0.8). Thus, 1,338 hits eventually remained as potential inhibitors of APE1.

We carried out molecular docking of the hits against APE1 to prioritize the hits using AutoDock Vina. Fig. 6 depicts the distribution of the predicted binding energies of the hits of the pharmacophore screen by docking. After molecular docking, we did not filter out compounds based on a particular threshold of the predicted value of the binding affinity but instead provide the top 10 hits in Fig. 7, their predicted binding poses in Supplementary Fig. 1, and all of the hits in Supplementary Table 1. This is because although Shityakov and Förster [25] reported that a compound having a binding affinity predicted by AutoDock Vina of lower than $-6 \mathrm{kcal} / \mathrm{mol}$ could be considered an active hit, the values are only predictive and rely on a somewhat empirical energy function. In other words, predicted binding affinities should be used restrictedly to help those who want to validate hits to determine the priority of subjects of an assay. Fig. 8 shows the alignments of the best hits into each pharmacophore model; all of the hits map well to the pharmacophore models. Of note, the rank of the docking results does not mean pharmacophore fitness, and all of the inhibitor compounds we found here can be mapped to the models well. The figure of pharmacophore alignment was made to provide an example showing that our hits can be mapped properly.

In summary, we screened more than 3 million lead-like compounds by pharmacophore modeling, and 1,338 hits were suggested to be potential inhibitors of APE1. However, this work has a limitation, due to the lack of experimental validation. Nevertheless, the list of hits in this work could reduce the time and cost of researchers who want to develop novel anticancer drugs inhibiting the activity of APE1, since we prioritized candidates of the experiments and since all of them have lead-like properties, which means that the hits are appropriate for further optimization and development into drugs.

Currently, there are several approaches that apply hits from a pharmacophore screen for further development in to a novel drug. Fei et al. [26] first developed a pharmacophore model of a drug target, like our method; then, 3D-quantitative structure-activity relationship (QSAR) modeling was used for validation and further virtual screening. Wieder et al. [27] proposed a novel approach combining pharmacophore modeling and molecular dynamics (MD) simulations, and they showed that their methods were likely to result in more robust hits. Like these approaches, the results from pharmacophore modeling could be adopted in other in silico methods, such as molecular docking, QSAR modeling, and MD simulation. It is worth combining these methods and our results to get more robust results. If further integrative approaches and in vitro or vivo assays of hits validate our
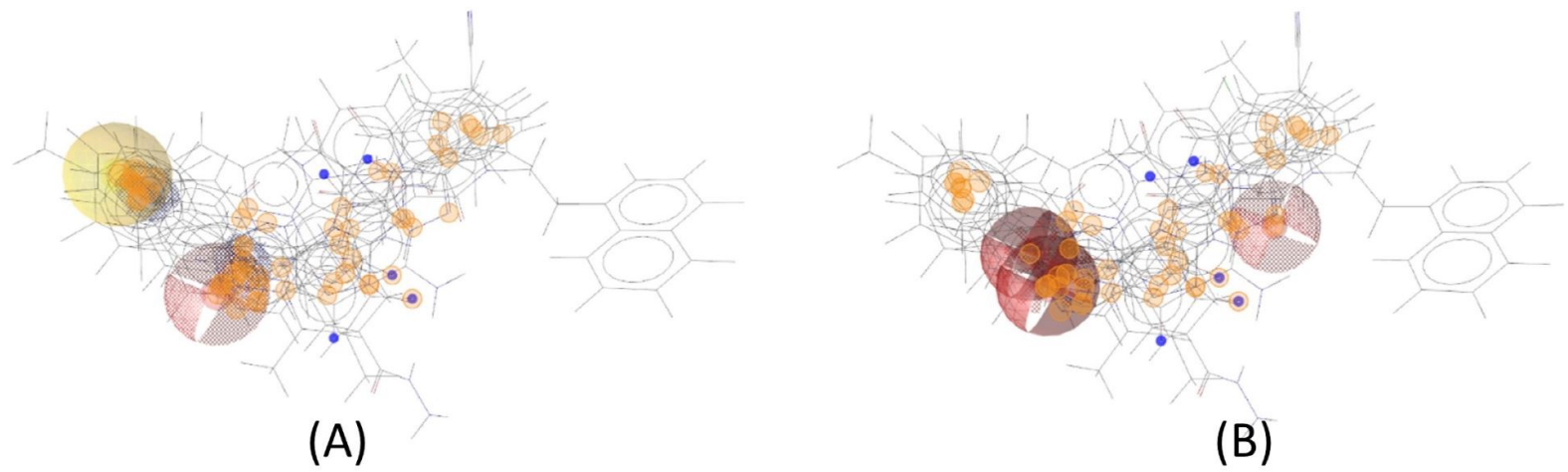

(B)

Fig. 8. Mapping of top 10 hits to pharmacophore models. (A) Model 1. (B) Model 2. 
results, our method could be applied to other drug targets, in addition to APE1.

ORCID: In Won Lee: http://orcid.org/0000-0002-4359-4 721; Jonghwan Yoon: http://orcid.org/0000-0002-3399-09 66; Gunhee Lee: http://orcid.org/0000-0001-7142-9072; Minho Lee: http://orcid.org/0000-0002-0168-9546

\section{Authors' contribution}

\author{
Conceptualization: ML \\ Data curation: IWL \\ Formal analysis: IWL, ML \\ Funding acquisition: ML \\ Methodology: IWL \\ Writing - original draft: IWL, JY, GL, ML \\ Writing - review and editing: ML
}

\section{Acknowledgments}

This work was supported by the National Research Foundation of Korea (NRF), funded by the Ministry of Science and ICT (NRF-2017R1C1B2008617 and NRF-2017M3A9B6061511), KREONET (Korea Research Environment Open NETwork) which is managed and operated by KISTI (Korea Institute of Science and Technology Information). In Won Lee was supported by a Sangji University scholarship for research assistants.

\section{Supplementary materials}

Supplementary data including one table and one figure can be found with this article online at http://www. genominfo.org/src/sm/gni-15-147-s001.pdf.

\section{References}

1. Bjorksten J, Acharya PV, Ashman S, Wetlaufer DB. Gerogenic fractions in the tritiated rat. J Am Geriatr Soc 1971;19:561-574.

2. Acharya PV. The isolation and partial characterization of age-correlated oligo-deoxyribo-ribonucleotides with covalently linked aspartyl-glutamyl polypeptides. Johns Hopkins Med J Suppl 1972;(1):254-260.

3. Barzilay G, Hickson ID. Structure and function of apurinic/apyrimidinic endonucleases. Bioessays 1995;17:713-719.

4. Xanthoudakis S, Smeyne RJ, Wallace JD, Curran T. The redox/DNA repair protein, Ref-1, is essential for early embryonic development in mice. Proc Natl Acad Sci U S A 1996; 93:8919-8923.

5. Ludwig DL, MacInnes MA, Takiguchi Y, Purtymun PE, Henrie $\mathrm{M}$, Flannery $\mathrm{M}$, et al. A murine AP-endonuclease gene-targeted deficiency with post-implantation embryonic progression and ionizing radiation sensitivity. Mutat Res 1998;409:17-29.

6. Kelley MR, Logsdon D, Fishel ML. Targeting DNA repair pathways for cancer treatment: what's new? Future Oncol 2014; 10:1215-1237.

7. Seiple LA, Cardellina JH 2nd, Akee R, Stivers JT. Potent inhibition of human apurinic/apyrimidinic endonuclease 1 by arylstibonic acids. Mol Pharmacol 2008;73:669-677.

8. Srinivasan A, Wang L, Cline CJ, Xie Z, Sobol RW, Xie XQ, et al. Identification and characterization of human apurinic/apyrimidinic endonuclease-1 inhibitors. Biochemistry 2012;51: 6246-6259.

9. Naidu MD, Agarwal R, Pena LA, Cunha L, Mezei M, Shen M, et al. Lucanthone and its derivative hycanthone inhibit apurinic endonuclease-1 (APE1) by direct protein binding. PLoS One 2011;6:e23679.

10. Simeonov A, Kulkarni A, Dorjsuren D, Jadhav A, Shen M, McNeill DR, et al. Identification and characterization of inhibitors of human apurinic/apyrimidinic endonuclease APE1. PLoS One 2009;4:e5740.

11. Luo M, Kelley MR. Inhibition of the human apurinic/apyrimidinic endonuclease (APE1) repair activity and sensitization of breast cancer cells to DNA alkylating agents with lucanthone. Anticancer Res 2004;24:2127-2134.

12. Liuzzi M, Talpaert-Borlé $M$. A new approach to the study of the base-excision repair pathway using methoxyamine.J Biol Chem 1985;260:5252-5258.

13. Fishel ML, Kelley MR. The DNA base excision repair protein Ape1/Ref-1 as a therapeutic and chemopreventive target. Mol Aspects Med 2007;28:375-395.

14. Wilson DM 3rd, Simeonov A. Small molecule inhibitors of DNA repair nuclease activities of APE1. Cell Mol Life Sci 2010;67:3621-3631.

15. Gaulton A, Bellis LJ, Bento AP, Chambers J, Davies M, Hersey A, et al. ChEMBL: a large-scale bioactivity database for drug discovery. Nucleic Acids Res 2012;40:D1100-D1107.

16. Wang Y, Bryant SH, Cheng T, Wang J, Gindulyte A, Shoemaker BA, et al. PubChem BioAssay: 2017 update. Nucleic Acids Res 2017;45:D955-D963.

17. Wolber G, Langer T. LigandScout: 3-D pharmacophores derived from protein-bound ligands and their use as virtual screening filters. J Chem Inf Model 2005;45:160-169.

18. Teague SJ, Davis AM, Leeson PD, Oprea T. The design of leadlike combinatorial libraries. Angew Chem Int Ed Engl 1999; 38:3743-3748.

19. Irwin JJ, Sterling T, Mysinger MM, Bolstad ES, Coleman RG. ZINC: a free tool to discover chemistry for biology. J Chem Inf Model 2012;52:1757-1768.

20. A decade of drug-likeness. Nat Rev Drug Discov 2007;6:853.

21. Lipinski CA, Lombardo F, Dominy BW, Feeney PJ. Experimental and computational approaches to estimate solubility and permeability in drug discovery and development settings. $A d v$ Drug Deliv Rev 2001;46:3-26.

22. Mol CD, Izumi T, Mitra S, Tainer JA. DNA-bound structures and mutants reveal abasic DNA binding by APE1 and DNA repair coordination [corrected]. Nature 2000;403:451-456.

23. Trott O, Olson AJ. AutoDock Vina: improving the speed and accuracy of docking with a new scoring function, efficient opti- 
mization, and multithreading. J Comput Chem 2010;31:455-461.

24. Le Guilloux V, Schmidtke P, Tuffery P. Fpocket: an open source platform for ligand pocket detection. BMC Bioinformatics 2009;10:168.

25. Shityakov S, Förster C. In silico predictive model to determine vector-mediated transport properties for the blood-brain barrier choline transporter. Adv Appl Bioinform Chem 2014;7:23-36.
26. Fei J, Zhou L, Liu T, Tang XY. Pharmacophore modeling, virtual screening, and molecular docking studies for discovery of novel Akt2 inhibitors. Int J Med Sci 2013;10:265-275.

27. Wieder M, Garon A, Perricone U, Boresch S, Seidel T, Almerico $\mathrm{AM}$, et al. Common hits approach: combining pharmacophore modeling and molecular dynamics simulations. J Chem Inf Model 2017;57:365-385. 


\section{SUPPLEMENTARY INFORMATION}

\section{Identification of New Potential APE1 Inhibitors by}

Pharmacophore Modeling and Molecular Docking

\section{In Won Lee ${ }^{1}$, Jonghwan Yoon ${ }^{2}$, Gunhee Lee ${ }^{2}$, Minho Lee $^{3 *}$}

${ }^{1}$ Department of Biological Science, Sangji University, Wonju 26339, Korea, ${ }^{2}$ Department of Biomedicine \& Health Sciences, Graduate School, The Catholic University of Korea, Seoul 06591, Korea, ${ }^{3}$ Catholic Precision Medicine Research Center, College of Medicine, The Catholic University of Korea, Seoul 06591, Korea 

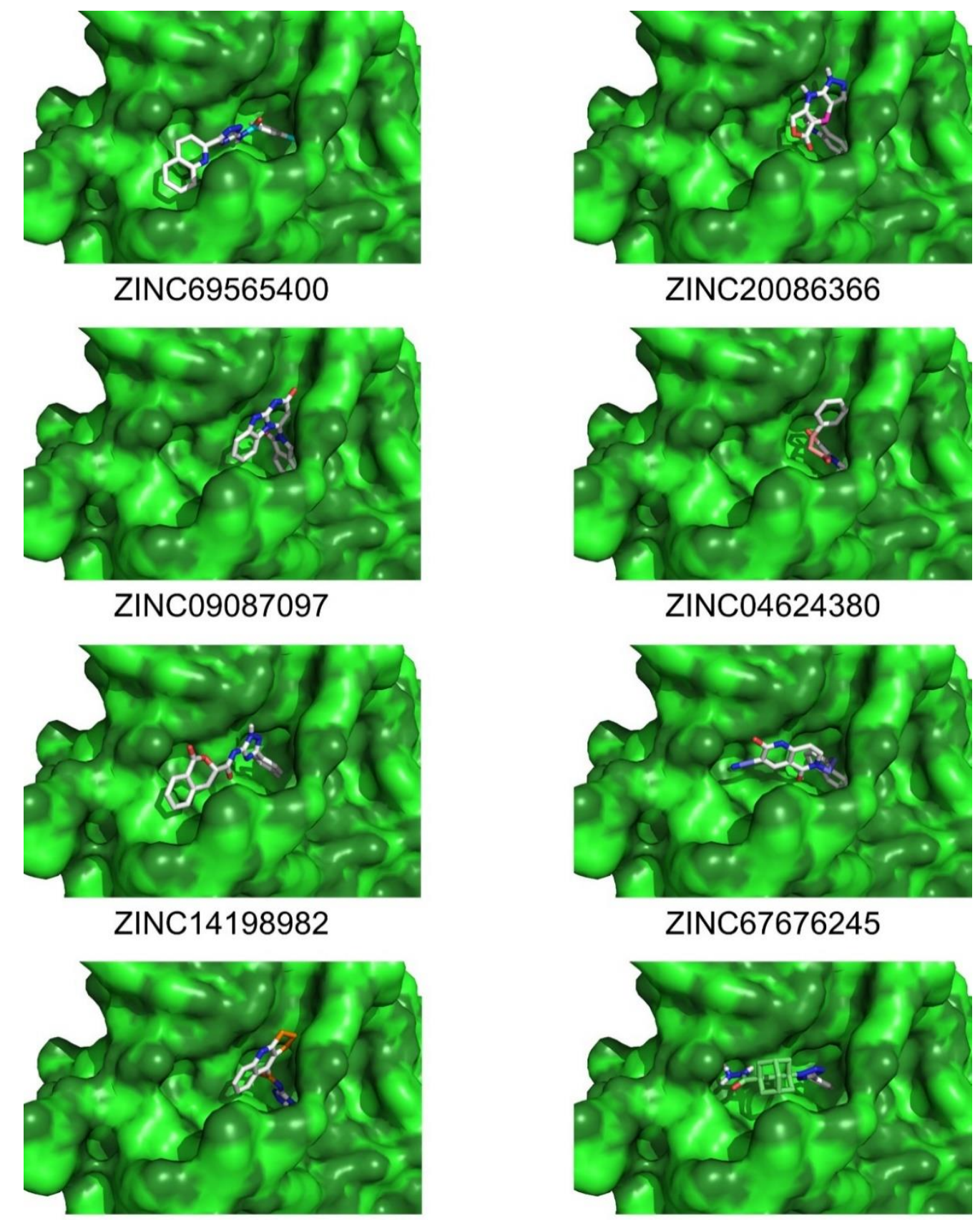

ZINC46083112

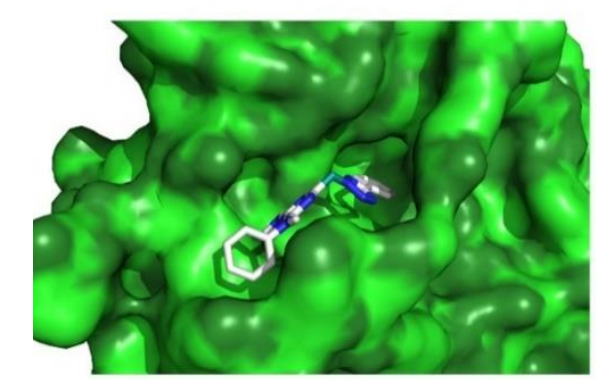

ZINC12533962

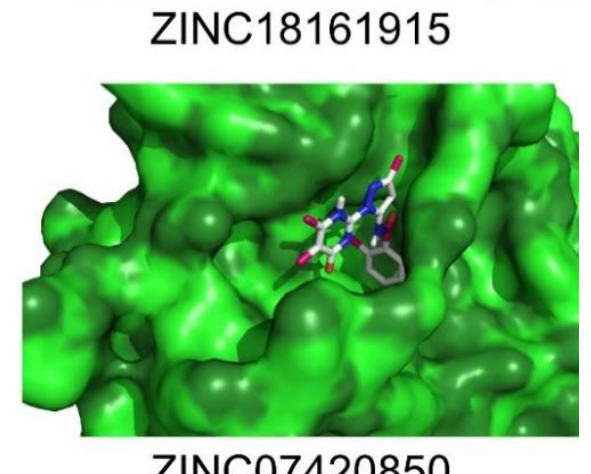

Supplementary Fig. 1. Predicted binding poses of the top 10 hits and AP endonuclease 1. 
Supplementary Table 1. List of 1,338 potential inhibitors of AP endonuclease 1 by pharmacophore screening and molecular docking

\begin{tabular}{|c|c|c|c|c|}
\hline ZINC ID & PubChem CID & $\begin{array}{l}\text { Pharmacophore } \\
\text { score } 1\end{array}$ & $\begin{array}{l}\text { Pharmacophore } \\
\text { score } 2\end{array}$ & $\begin{array}{l}\text { Binding } \\
\text { affinity }\end{array}$ \\
\hline ZINC69565400 & 53580594 & 41.00639 & 43.377747 & -8.7 \\
\hline ZINC20086366 & $51603501,28611523,25248999$ & 40.193176 & 44.288307 & -8.6 \\
\hline ZINC09087097 & 4909512,40928637 & 40.654366 & 43.33812 & -8.6 \\
\hline ZINC04624380 & 5299120,7305393 & 40.906654 & 44.409916 & -8.6 \\
\hline ZINC14198982 & 17568456,25987655 & 40.984146 & 42.76821 & -8.5 \\
\hline ZINC67676245 & 56712240 & 39.836067 & 43.321037 & -8.5 \\
\hline ZINC46083122 & 51113769 & 40.194237 & 43.284782 & -8.4 \\
\hline ZINC18161915 & 843225,3300507 & 41.150127 & 44.36736 & -8.4 \\
\hline ZINC12533962 & 17448744 & 41.15087 & 43.60126 & -8.4 \\
\hline ZINC07420850 & 51430633,8516743 & 39.675964 & 43.53222 & -8.4 \\
\hline ZINC07646218 & 8752998 & 41.03507 & 43.322594 & -8.4 \\
\hline ZINC22263860 & 24698181 & 40.134243 & 45.798782 & -8.3 \\
\hline ZINC28716248 & 35873053,35873054 & 40.797756 & 43.46943 & -8.3 \\
\hline ZINC16956851 & 27444029 & 40.977768 & 43.403572 & -8.3 \\
\hline ZINC00032557 & 582551 & 41.26294 & 45.560642 & -8.3 \\
\hline ZINC09691166 & 17407752,9246897 & 40.98685 & 44.572247 & -8.3 \\
\hline ZINC71803297 & 56825790 & 41.01689 & 42.84125 & -8.3 \\
\hline ZINC03900862 & 2849543,51397836 & 40.186153 & 43.359375 & -8.2 \\
\hline ZINC19373000 & 51590423,46973619 & 41.066746 & 44.160034 & -8.2 \\
\hline ZINC07813720 & 8933276,16339923 & 40.81163 & 45.81323 & -8.2 \\
\hline ZINC00081470 & 4195018,700996 & 40.310978 & 44.68419 & -8.2 \\
\hline ZINC67677150 & 50966999 & 40.068657 & 43.8693 & -8.1 \\
\hline ZINC48252179 & 47058741 & 41.00527 & 44.103455 & -8.1 \\
\hline ZINC20086368 & 28611526,25248999 & 40.192806 & 44.28888 & -8.1 \\
\hline ZINC01857460 & 51382095 & 41.132175 & 42.971542 & -8.1 \\
\hline ZINC67822168 & 53599765 & 41.00122 & 43.107483 & -8 \\
\hline ZINC05730179 & 5310761 & 39.995228 & 43.90605 & -8 \\
\hline ZINC06504095 & 5638632,6088957 & 41.160614 & 43.867977 & -8 \\
\hline ZINC08581318 & 220884 & 39.94408 & 44.172806 & -8 \\
\hline ZINC07333877 & 8425776,16327956 & 41.093735 & 44.574345 & -8 \\
\hline ZINC02527998 & 2736033,2736032 & 41.14576 & 45.193874 & -8 \\
\hline ZINC57819878 & 48927451 & 41.00126 & 43.93179 & -8 \\
\hline ZINC06863616 & 4128582 & 40.309692 & 43.244865 & -8 \\
\hline ZINC72221331 & 66485109 & 40.97538 & 43.520397 & -7.9 \\
\hline ZINC78681112 & 60309163 & 41.018013 & 43.91385 & -7.9 \\
\hline ZINC16311417 & 27055998,43835904 & 40.565964 & 45.563713 & -7.9 \\
\hline ZINC01395174 & 1481964 & 39.97632 & 44.54124 & -7.9 \\
\hline ZINC04807850 & $\begin{array}{l}3428894,44660697,51407295, \\
7365510\end{array}$ & 40.595795 & 44.423622 & -7.9 \\
\hline ZINC71762022 & 56877417 & 41.115467 & 43.77193 & -7.9 \\
\hline ZINC07333875 & 8425778,16327956 & 41.09811 & 44.19793 & -7.9 \\
\hline ZINC69808716 & 53606664 & 41.01794 & 43.081497 & -7.9 \\
\hline ZINC07333876 & 8425779,16327956 & 41.09301 & 44.57296 & -7.9 \\
\hline ZINC09691167 & 17407752,9246898 & 40.988075 & 44.57168 & -7.9 \\
\hline ZINC06887422 & 20914791 & 40.871284 & 44.63051 & -7.9 \\
\hline ZINC67340583 & 53337125 & 39.759563 & 44.01888 & -7.9 \\
\hline ZINC71753018 & 56873732 & 40.78277 & 43.847424 & -7.9 \\
\hline ZINC04624382 & 5299120,7305394 & 40.90664 & 43.99303 & -7.9 \\
\hline ZINC08776545 & 17550747 & 40.805424 & 45.29248 & -7.9 \\
\hline ZINC36143324 & 7336024 & 41.424095 & 44.4535 & -7.8 \\
\hline ZINC66766711 & 56803748 & 39.99118 & 43.602165 & -7.8 \\
\hline
\end{tabular}




\begin{tabular}{|c|c|c|c|c|}
\hline ZINC44891099 & 52208404,47076168 & 39.61378 & 44.165123 & -7.8 \\
\hline ZINC05196641 & $40511903,54750067,54682459$ & 39.85478 & 43.98333 & -7.8 \\
\hline ZINC22521090 & $30389525,7072922,4302482$ & 41.19613 & 45.108246 & -7.8 \\
\hline ZINC16430870 & $51561978,4055501,27126693$ & 39.844334 & 44.35685 & -7.8 \\
\hline ZINC06654288 & 8026484 & 41.203903 & 44.80751 & -7.8 \\
\hline ZINC21984806 & 29921482 & 39.875546 & 43.64945 & -7.8 \\
\hline ZINC72159403 & 56908689 & 41.089214 & 43.71085 & -7.8 \\
\hline ZINC16956225 & 44172473,1402151 & 39.864803 & 44.71934 & -7.8 \\
\hline ZINC75519126 & 53163470 & 39.60385 & 44.24327 & -7.8 \\
\hline ZINC01401802 & 51380285,1487349 & 40.055305 & 43.39957 & -7.8 \\
\hline ZINC22144786 & 30048505,42934235 & 40.994324 & 44.238327 & -7.8 \\
\hline ZINC49474313 & 53215490 & 40.822304 & 44.06403 & -7.8 \\
\hline ZINC66932039 & 56784604 & 41.01031 & 42.59468 & -7.8 \\
\hline ZINC06691181 & $\begin{array}{l}4152693,6022536,54722165, \\
40634935\end{array}$ & 40.116154 & 43.099125 & -7.8 \\
\hline ZINC23369363 & 31045060,31045059 & 41.07701 & 43.28105 & -7.8 \\
\hline ZINC29785373 & 42955724,37028723 & 41.007656 & 44.88097 & -7.8 \\
\hline ZINC17058894 & 949493,44172610 & 39.864628 & 42.547577 & -7.8 \\
\hline ZINC35378115 & 53004370 & 40.524597 & 42.976257 & -7.8 \\
\hline ZINC18196258 & 24216321 & 41.3786 & 44.321587 & -7.7 \\
\hline ZINC03131441 & 44722272,3410945 & 40.470627 & 45.21289 & -7.7 \\
\hline ZINC66766713 & 56803748 & 39.991924 & 43.35018 & -7.7 \\
\hline ZINC03513094 & 2630165 & 41.131363 & 44.377697 & -7.7 \\
\hline ZINC58315777 & 51096570 & 41.13335 & 45.29671 & -7.7 \\
\hline ZINC65534404 & 53512254 & 41.133167 & 42.454556 & -7.7 \\
\hline ZINC71805845 & 60226840 & 40.87792 & 45.55764 & -7.7 \\
\hline ZINC32754192 & 39976464 & 40.134254 & 43.196632 & -7.7 \\
\hline ZINC13408043 & 5705854 & 41.15726 & 44.54155 & -7.7 \\
\hline ZINC20085974 & 28611005,25248927 & 39.3119 & 44.42549 & -7.7 \\
\hline ZINC18207737 & 6790402 & 41.293533 & 43.727184 & -7.7 \\
\hline ZINC65531442 & 53512008 & 41.243206 & 43.608036 & -7.7 \\
\hline ZINC00612801 & 972533 & 39.99617 & 44.211422 & -7.7 \\
\hline ZINC20178305 & 22432895 & 41.25519 & 43.906235 & -7.7 \\
\hline ZINC35316024 & 43813803 & 40.17817 & 44.20072 & -7.7 \\
\hline ZINC69439631 & 53525028 & 40.882217 & 45.753693 & -7.7 \\
\hline ZINC01235377 & $\begin{array}{l}28384577,5421439,931772, \\
6955375\end{array}$ & 41.060184 & 45.47464 & -7.7 \\
\hline ZINC72411990 & 56918506 & 39.087776 & 44.9308 & -7.7 \\
\hline ZINC06025124 & 51418924,4049388 & 41.330532 & 43.46222 & -7.7 \\
\hline ZINC09644457 & 51466717,16343127 & 41.278343 & 44.65683 & -7.7 \\
\hline ZINC69771759 & 53603406 & 40.891357 & 44.664906 & -7.7 \\
\hline ZINC44870230 & 52207395,45796266 & 39.961792 & 43.564117 & -7.7 \\
\hline ZINC49549982 & 52322357,51054441 & 40.825718 & 45.815525 & -7.7 \\
\hline ZINC08394170 & 9507919,16305469 & 41.16206 & 43.38234 & -7.6 \\
\hline ZINC59327554 & 52659678 & 41.405247 & 43.098183 & -7.6 \\
\hline ZINC83237297 & 53270550 & 39.049408 & 43.761127 & -7.6 \\
\hline ZINC13218483 & $4055501,27126694,51519309$ & 39.844334 & 44.13523 & -7.6 \\
\hline ZINC07320631 & 40687663 & 39.627487 & 44.506638 & -7.6 \\
\hline ZINC17781489 & 27739430 & 41.21642 & 44.318535 & -7.6 \\
\hline ZINC71762752 & 56877938 & 40.18908 & 43.29615 & -7.6 \\
\hline ZINC03425414 & 2544764 & 41.38584 & 43.908615 & -7.6 \\
\hline ZINC06504096 & 5638631,6088957 & 41.160027 & 43.871574 & -7.6 \\
\hline ZINC70461283 & 56772294 & 41.015846 & 43.469078 & -7.6 \\
\hline ZINC70461284 & 56772294 & 41.01586 & 43.093765 & -7.6 \\
\hline ZINC65153401 & 53143381 & 41.05384 & 44.429695 & -7.6 \\
\hline ZINC15930288 & 51552329,19576482 & 40.93067 & 46.056007 & -7.6 \\
\hline ZINC01061496 & 6978104,1251530 & 40.512447 & 45.630737 & -7.6 \\
\hline
\end{tabular}




\begin{tabular}{|c|c|c|c|c|}
\hline ZINC05546951 & $51414439,23137730,6834166$ & 40.94207 & 43.444748 & -7.6 \\
\hline ZINC36028896 & 5002078,7276493 & 41.141075 & 44.7101 & -7.6 \\
\hline ZINC72465916 & 66504699 & 41.118916 & 44.030632 & -7.6 \\
\hline ZINC32106138 & 39354101 & 40.82926 & 43.105896 & -7.6 \\
\hline ZINC34872852 & $2442106,7042007,2442107$ & 40.80655 & 45.435627 & -7.6 \\
\hline ZINC49882309 & 63898678 & 40.83666 & 43.48653 & -7.6 \\
\hline ZINC48253576 & 52284503,47014262 & 41.06247 & 43.550453 & -7.6 \\
\hline ZINC25377520 & 32687412,47008435 & 40.078506 & 43.23179 & -7.6 \\
\hline ZINC04270620 & 11887288 & 40.544815 & 44.175762 & -7.6 \\
\hline ZINC69704510 & 53597246 & 40.060265 & 43.50159 & -7.6 \\
\hline ZINC58393031 & 51101115 & 39.882553 & 44.362553 & -7.6 \\
\hline ZINC72319655 & 56973351 & 40.49263 & 45.659306 & -7.6 \\
\hline ZINC44891101 & 52208405,47076168 & 39.61378 & 43.276375 & -7.6 \\
\hline ZINC29785372 & 42955724,37028722 & 41.00779 & 44.63262 & -7.6 \\
\hline ZINC69702473 & 53596589 & 41.000557 & 44.375114 & -7.6 \\
\hline ZINC83237290 & 53270550 & 39.049408 & 44.347755 & -7.6 \\
\hline ZINC83237295 & 53270550 & 39.049408 & 45.88568 & -7.5 \\
\hline ZINC00044740 & $5391741,6920639,5908098$ & 39.984444 & 44.505875 & -7.5 \\
\hline ZINC72427217 & 56896425 & 41.14916 & 44.985542 & -7.5 \\
\hline ZINC13534350 & 25794346,18524185 & 40.678276 & 43.388363 & -7.5 \\
\hline ZINC26537568 & 33811761 & 40.997852 & 42.873775 & -7.5 \\
\hline ZINC65501997 & 53541513 & 41.245785 & 44.436363 & -7.5 \\
\hline ZINC12986880 & 25651036,25651035 & 41.217224 & 44.238823 & -7.5 \\
\hline ZINC31777062 & 51655764,45790190 & 40.17057 & 44.35639 & -7.5 \\
\hline ZINC19093887 & $3857607,28065418,5940993$ & 40.42855 & 43.943974 & -7.5 \\
\hline ZINC11335481 & 51485512,41929670 & 40.937237 & 43.327835 & -7.5 \\
\hline ZINC08770629 & 8073089 & 40.93525 & 43.175148 & -7.5 \\
\hline ZINC78681110 & 60309163 & 41.016647 & 43.91416 & -7.5 \\
\hline ZINC49543749 & 52320296,51052377 & 39.69057 & 43.47857 & -7.5 \\
\hline ZINC31952552 & 39168582,47013790 & 39.960457 & 44.370815 & -7.5 \\
\hline ZINC69439634 & 53525028 & 40.882515 & 45.747105 & -7.5 \\
\hline ZINC13126580 & $\begin{array}{l}\text { 6743377,5732279,51517545, } \\
5407898\end{array}$ & 41.215473 & 43.44103 & -7.5 \\
\hline ZINC65459220 & 50974304 & 39.871807 & 44.40284 & -7.5 \\
\hline ZINC04099168 & $\begin{array}{l}44152247,152936,40473225, \\
42629338,114851\end{array}$ & 40.05093 & 44.681107 & -7.5 \\
\hline ZINC05343683 & 51412169 & 41.16346 & 43.1354 & -7.5 \\
\hline ZINC05343132 & 40528217,3726659 & 41.12213 & 45.32207 & -7.5 \\
\hline ZINC05927382 & 941673,40572273 & 41.26936 & 43.012653 & -7.5 \\
\hline ZINC71917919 & 60456982 & 40.569008 & 45.30564 & -7.5 \\
\hline ZINC23928128 & 46613854,31426117 & 40.728596 & 44.176636 & -7.5 \\
\hline ZINC65565360 & 53546864 & 39.90771 & 43.622303 & -7.4 \\
\hline ZINC04938661 & 51408226,2815909 & 41.11892 & 43.382805 & -7.4 \\
\hline ZINC08156622 & 51432197,9291945 & 41.27617 & 44.118362 & -7.4 \\
\hline ZINC65536339 & 53544051 & 39.908573 & 43.621216 & -7.4 \\
\hline ZINC04523150 & $\begin{array}{l}24186039,54609189,80539, \\
23679260\end{array}$ & 39.756577 & 45.47775 & -7.4 \\
\hline ZINC19624503 & 28246393 & 41.00454 & 43.343258 & -7.4 \\
\hline ZINC40267557 & 51971836,49653869 & 40.914597 & 44.09568 & -7.4 \\
\hline ZINC35615770 & 45600204 & 40.860203 & 44.383553 & -7.4 \\
\hline ZINC40168661 & 43828475 & 39.909695 & 43.191624 & -7.4 \\
\hline ZINC67677494 & 56712427 & 40.13364 & 43.81382 & -7.4 \\
\hline ZINC68945842 & 60285729 & 41.028343 & 44.000885 & -7.4 \\
\hline ZINC32951131 & 46507241 & 41.159805 & 43.33236 & -7.4 \\
\hline ZINC67691480 & 56750090 & 40.80662 & 43.318573 & -7.4 \\
\hline ZINC16948987 & 6836689 & 41.29487 & 43.273575 & -7.4 \\
\hline ZINC40176455 & 46398289 & 41.342216 & 44.01334 & -7.4 \\
\hline
\end{tabular}




\begin{tabular}{|c|c|c|c|c|}
\hline ZINC72406767 & 56881337 & 39.784515 & 45.452354 & -7.4 \\
\hline ZINC36360552 & 51720714,43865679 & 40.151867 & 43.19236 & -7.4 \\
\hline ZINC72171319 & 56916329 & 41.131294 & 44.99334 & -7.4 \\
\hline ZINC18068125 & 6553413,2972795 & 41.05159 & 43.242897 & -7.4 \\
\hline ZINC69628340 & 53589089 & 41.016895 & 42.74201 & -7.4 \\
\hline ZINC58229872 & 49079637 & 40.93766 & 44.30857 & -7.4 \\
\hline ZINC69704513 & 53597246 & 40.059242 & 43.23833 & -7.4 \\
\hline ZINC65502000 & 53541513 & 41.246273 & 44.51023 & -7.4 \\
\hline ZINC23702513 & $9070766,9070767,16281755$ & 40.245697 & 43.68691 & -7.4 \\
\hline ZINC65364163 & 47001650 & 40.79735 & 43.3219 & -7.4 \\
\hline ZINC69534072 & 53577322 & 41.38813 & 43.239357 & -7.4 \\
\hline ZINC13718975 & 17599170 & 40.958286 & 44.927822 & -7.4 \\
\hline ZINC01609684 & 1549997,261602 & \begin{tabular}{|l|l|}
39.697403 \\
\end{tabular} & 45.07562 & -7.4 \\
\hline ZINC71279179 & 53370416 & 41.283367 & 43.90082 & -7.4 \\
\hline ZINC06007299 & 6789735 & 40.614956 & 43.31456 & -7.4 \\
\hline ZINC38828821 & 43864914 & 40.65043 & 44.050594 & -7.4 \\
\hline ZINC22144783 & 30048503,42934235 & 40.99655 & 44.34017 & -7.4 \\
\hline ZINC48230720 & 58896227 & 40.30384 & 44.50443 & -7.4 \\
\hline ZINC33403397 & $25728995,5726001,2867539$ & 41.143127 & 43.60938 & -7.4 \\
\hline ZINC12781078 & 24235884,25506339 & 41.13242 & 43.156246 & -7.4 \\
\hline ZINC23702509 & $9070756,9070757,16281755$ & 40.244858 & 43.6995 & -7.4 \\
\hline ZINC08914253 & 40870414,6464023 & 39.6896 & 44.602825 & -7.4 \\
\hline ZINC04978088 & $3739063,1387091,11904425$ & 39.836952 & 43.299004 & -7.4 \\
\hline ZINC00383020 & $848639,2952912,6949779$ & 41.385544 & 43.76314 & -7.4 \\
\hline ZINC49454193 & 53213210 & 41.320656 & 42.405052 & -7.4 \\
\hline ZINC69454913 & 53567078 & 41.158733 & 44.18637 & -7.4 \\
\hline ZINC49453625 & 53212749 & 41.11808 & 44.80846 & -7.4 \\
\hline ZINC49453624 & 53212749 & 41.11808 & 45.3218 & -7.4 \\
\hline ZINC14135091 & 17449437 & 40.284683 & 44.21864 & -7.4 \\
\hline ZINC58282353 & 51100060 & 41.191456 & 44.235695 & -7.3 \\
\hline ZINC71924190 & 56795625 & 41.244 & 43.82785 & -7.3 \\
\hline ZINC20721587 & 29130895,25239789 & 41.147133 & 44.891365 & -7.3 \\
\hline ZINC19596400 & $68146242,68459,1563313$ & 40.006042 & 44.123257 & -7.3 \\
\hline ZINC65506337 & 50980826 & 40.88359 & 45.799744 & -7.3 \\
\hline ZINC71893470 & 56786219 & 40.559967 & 43.933235 & -7.3 \\
\hline ZINC12428208 & 5092643,714067 & 40.99134 & 45.531933 & -7.3 \\
\hline ZINC11692954 & 42109034,17015160 & 40.68804 & 45.76515 & -7.3 \\
\hline ZINC13534359 & 18524185 & 40.67697 & 43.288826 & -7.3 \\
\hline ZINC05060857 & 2877377 & 41.134308 & 43.46235 & -7.3 \\
\hline ZINC19890480 & 2229603,51602378 & 41.162224 & 45.36945 & -7.3 \\
\hline ZINC71916552 & 56794497 & 39.906 & 44.02619 & -7.3 \\
\hline ZINC05846195 & $\begin{array}{l}51417368,18650740,56606124, \\
84592,66848758 \\
\end{array}$ & 40.188347 & 44.94799 & -7.3 \\
\hline ZINC67677493 & 56712427 & 40.134716 & 43.96515 & -7.3 \\
\hline ZINC02312130 & $932225,25806303,5421619$ & 41.282295 & 44.01233 & -7.3 \\
\hline ZINC32918485 & 44122285 & 40.963417 & 43.8796 & -7.3 \\
\hline ZINC39124464 & 13112787 & 40.443947 & 43.19419 & -7.3 \\
\hline ZINC69352224 & 53556588 & 40.900238 & 45.29671 & -7.3 \\
\hline ZINC04727792 & 5298798,7335559 & 40.969193 & 45.67144 & -7.3 \\
\hline ZINC01514884 & 1548505 & 41.146107 & 45.4972 & -7.3 \\
\hline ZINC18089972 & 51577709,849716 & 41.021442 & 42.502483 & -7.3 \\
\hline ZINC13657823 & 2816447,51525574 & 41.23385 & 44.76842 & -7.3 \\
\hline ZINC33285939 & 703503 & 40.074368 & 44.191757 & -7.3 \\
\hline ZINC04158751 & $6848401,51400204,16805410$ & 41.14508 & 43.660355 & -7.3 \\
\hline ZINC71405221 & 53469875 & 40.8056 & 44.888298 & -7.3 \\
\hline ZINC19843144 & $5663271,6757261,5506279$ & 40.43435 & 44.49781 & -7.3 \\
\hline ZINC53114913 & 53512413 & 41.139374 & 45.252674 & -7.3 \\
\hline
\end{tabular}




\begin{tabular}{|c|c|c|c|c|}
\hline ZINC25174997 & 6762884 & 41.203346 & 43.93299 & -7.3 \\
\hline ZINC06509043 & 4963779 & 41.218887 & 45.906578 & -7.3 \\
\hline ZINC20541542 & 43824748 & 41.149155 & 45.223343 & -7.3 \\
\hline ZINC08764859 & 54715632 & 40.135017 & 43.592644 & -7.3 \\
\hline ZINC13569875 & 6884054,932225 & 41.288567 & 43.89179 & -7.3 \\
\hline ZINC08578980 & 40773380,2323920 & 39.659256 & 43.11826 & -7.3 \\
\hline ZINC05516877 & \begin{tabular}{|l|}
$21781258,3718559,44888627$ \\
\end{tabular} & 41.29416 & 44.510822 & -7.3 \\
\hline ZINC04749669 & $5391742,7346410,5908098$ & 39.98338 & 43.055935 & -7.3 \\
\hline ZINC64920009 & 53068055 & 40.95331 & 44.65331 & -7.3 \\
\hline ZINC20721590 & 29130897,25239789 & 41.167515 & 44.825882 & -7.3 \\
\hline ZINC33315964 & 5415573,885056 & 41.140312 & 44.540207 & -7.3 \\
\hline ZINC05072586 & 7481747,3502412 & 40.003647 & 45.61891 & -7.3 \\
\hline ZINC55271764 & 52392033,46992856 & 41.124245 & 44.900223 & -7.3 \\
\hline ZINC46494405 & 56796159 & 41.4467 & 44.488075 & -7.3 \\
\hline ZINC32918480 & 44122283 & 41.001938 & 43.8795 & -7.3 \\
\hline ZINC35314039 & 43814849 & 40.8914 & 45.57711 & -7.3 \\
\hline ZINC12340662 & 42545239 & 41.15964 & 44.140495 & -7.3 \\
\hline ZINC80339460 & 60180720 & 40.87528 & 44.837955 & -7.3 \\
\hline ZINC13957338 & \begin{tabular}{|l|l|}
$40547512,6096694,25904408$ \\
\end{tabular} & 40.55928 & 43.384056 & -7.3 \\
\hline ZINC68576737 & 54594625 & 41.006046 & 43.07414 & -7.3 \\
\hline ZINC00110567 & 714806 & 41.252193 & 43.598175 & -7.3 \\
\hline ZINC08606821 & 51436271,662330 & 40.3836 & 43.436512 & -7.3 \\
\hline ZINC25464077 & 51648636,24654119 & 40.88159 & 45.60512 & -7.3 \\
\hline ZINC55107949 & 52382416,46985864 & 41.32705 & 44.983948 & -7.3 \\
\hline ZINC71847183 & 56826386 & 41.153625 & 43.822865 & -7.3 \\
\hline ZINC65530975 & 50984410 & 41.051716 & 43.725353 & -7.2 \\
\hline ZINC57963958 & 47093590 & 40.26156 & 44.409912 & -7.2 \\
\hline ZINC06414288 & 3651766,44167330 & 41.147858 & 44.577415 & -7.2 \\
\hline ZINC65886545 & 56799607 & 39.603195 & 44.343067 & -7.2 \\
\hline ZINC67284641 & 50929318 & 40.160763 & 44.205032 & -7.2 \\
\hline ZINC05243833 & 1498344,40517181 & 41.158035 & 43.880875 & -7.2 \\
\hline ZINC11568101 & \begin{tabular}{|l}
24252750,42034855 \\
\end{tabular} & 40.050114 & 45.64509 & -7.2 \\
\hline ZINC40165640 & 46397573 & 39.58171 & 44.96981 & -7.2 \\
\hline ZINC59402585 & 5415570,884942 & 41.210533 & 44.18254 & -7.2 \\
\hline ZINC48229542 & 47058739 & 40.23667 & 43.79521 & -7.2 \\
\hline ZINC10901020 & 41719281 & 41.1396 & 45.553802 & -7.2 \\
\hline ZINC15108171 & 26434595,42647526 & 40.844433 & 45.305656 & -7.2 \\
\hline ZINC17304990 & \begin{tabular}{|l|}
24539599,51572742 \\
\end{tabular} & 40.99888 & 42.87452 & -7.2 \\
\hline ZINC45574528 & 52219932,46652850 & 40.799107 & 43.562897 & -7.2 \\
\hline ZINC55358227 & 52396748,50976128 & 40.187294 & 44.41239 & -7.2 \\
\hline ZINC12756814 & 25487567,25487566 & 41.217377 & 44.649235 & -7.2 \\
\hline ZINC00381943 & \begin{tabular}{|l|l|}
6949739,847878 \\
\end{tabular} & 41.291515 & 44.41197 & -7.2 \\
\hline ZINC20272380 & 28811515 & 40.993935 & 44.659676 & -7.2 \\
\hline ZINC33365807 & 5620665,2006170 & 39.702602 & 44.242016 & -7.2 \\
\hline ZINC39124463 & 13112787 & 40.444603 & 43.19444 & -7.2 \\
\hline ZINC08830265 & 2355923,9586573 & 39.98758 & 42.816963 & -7.2 \\
\hline ZINC05204198 & 7553601 & 40.670578 & 44.102848 & -7.2 \\
\hline ZINC35669014 & 4771079 & 41.129765 & 45.348347 & -7.2 \\
\hline ZINC12804681 & 16582453 & 41.022575 & 45.785656 & -7.2 \\
\hline ZINC04061683 & $7086533,6544382,2952912$ & 41.386913 & 43.330666 & -7.2 \\
\hline ZINC67788840 & 56715579 & 39.83471 & 43.318863 & -7.2 \\
\hline ZINC02775311 & \begin{tabular}{|l|l|}
$27153747,19622675,51389922$ \\
\end{tabular} & 40.96011 & 45.392788 & -7.2 \\
\hline ZINC71907095 & 60451424 & 41.13263 & 42.86867 & -7.2 \\
\hline ZINC38768026 & 51897053,45513931 & 39.865585 & 42.610165 & -7.2 \\
\hline ZINC41076439 & 52095971 & 41.167526 & 43.57198 & -7.2 \\
\hline ZINC39125357 & 12399589 & 40.96917 & 43.542896 & -7.2 \\
\hline ZINC72152098 & 56894782 & 41.113705 & 44.562923 & -7.2 \\
\hline
\end{tabular}




\begin{tabular}{|c|c|c|c|c|}
\hline ZINC19972653 & $\begin{array}{l}3534099,54676721,28503102, \\
54676436,56684091,44659827, \\
758909,54741226,45053318\end{array}$ & 40.174828 & 42.81953 & -7.2 \\
\hline ZINC38162173 & 6791453 & 41.133793 & 44.70314 & -7.2 \\
\hline ZINC27562009 & 42926268,34924350 & 41.38498 & 43.267822 & -7.2 \\
\hline ZINC67321046 & 51370236 & 39.492725 & 43.179813 & -7.2 \\
\hline ZINC10339352 & 13562808 & 39.93637 & 43.62852 & -7.2 \\
\hline ZINC33048721 & 46346358 & 39.557568 & 43.878086 & -7.2 \\
\hline ZINC32598900 & 39841833 & 40.634354 & 44.281563 & -7.2 \\
\hline ZINC31997562 & 39226683 & 40.781845 & 45.307137 & -7.2 \\
\hline ZINC00479749 & 900702 & 40.02168 & 44.0288 & -7.2 \\
\hline ZINC69775585 & 53604524 & 41.01733 & 43.079758 & -7.2 \\
\hline ZINC36533680 & 51777314,45933638 & 41.294502 & 46.052628 & -7.2 \\
\hline ZINC49544109 & $53371341,52320414,51052577$ & 40.997105 & 44.438198 & -7.2 \\
\hline ZINC69486628 & 53570044 & 39.60428 & 43.69329 & -7.2 \\
\hline ZINC69173286 & 53500936 & 40.976643 & 44.638275 & -7.2 \\
\hline ZINC38488060 & 44070597 & 39.58695 & 44.635906 & -7.2 \\
\hline ZINC72417008 & 66490338 & 40.811405 & 44.658062 & -7.2 \\
\hline ZINC31648445 & 38869404,47006626 & 40.97959 & 44.0261 & -7.2 \\
\hline ZINC71328509 & 53367334 & 40.98262 & 43.65821 & -7.2 \\
\hline ZINC04205234 & 4961888,7128671 & 40.244007 & 43.88365 & -7.2 \\
\hline ZINC67284399 & 50929241 & 40.577072 & 44.39699 & -7.2 \\
\hline ZINC12974036 & 25639454 & 41.149345 & 43.093185 & -7.2 \\
\hline ZINC00034330 & 672532 & 39.99184 & 44.285416 & -7.2 \\
\hline ZINC06129896 & 1965930,4898621 & 41.05349 & 43.624535 & -7.2 \\
\hline ZINC34465562 & 10781314 & 40.13139 & 42.71784 & -7.2 \\
\hline ZINC31952556 & 39168586,47013790 & 39.960457 & 44.317482 & -7.2 \\
\hline ZINC06252590 & 6785751,6411365 & 41.321716 & 44.487656 & -7.2 \\
\hline ZINC39772733 & 19256575 & 41.038162 & 42.69838 & -7.2 \\
\hline ZINC58345495 & 18137332 & 40.215977 & 43.488625 & -7.2 \\
\hline ZINC41005691 & 52083294 & 41.132175 & 43.71231 & -7.2 \\
\hline ZINC02348350 & 1961458 & 41.157898 & 45.85177 & -7.1 \\
\hline ZINC16778623 & 27331690,16443333 & 40.00354 & 45.531437 & -7.1 \\
\hline ZINC69921397 & 53617621 & 40.21124 & 44.82035 & -7.1 \\
\hline ZINC72158041 & 56907928 & 41.130695 & 43.141396 & -7.1 \\
\hline ZINC69445224 & 53564593 & 39.770157 & 42.972294 & -7.1 \\
\hline ZINC06497742 & 5688597,40619658 & 40.96643 & 43.77597 & -7.1 \\
\hline ZINC00109697 & 2740544,51372363 & 40.998196 & 44.207294 & -7.1 \\
\hline ZINC33313953 & 20873537 & 40.174637 & 44.06584 & -7.1 \\
\hline ZINC12417917 & 5306165,7350523 & 41.251663 & 45.826546 & -7.1 \\
\hline ZINC03772546 & 2722554 & 41.26451 & 45.06756 & -7.1 \\
\hline ZINC71945507 & 60493107 & 39.963562 & 43.31019 & -7.1 \\
\hline ZINC69813907 & 53608265 & 41.147125 & 45.106834 & -7.1 \\
\hline ZINC61719107 & 52899400 & 41.078262 & 43.93142 & -7.1 \\
\hline ZINC49452968 & 53212249 & 40.203667 & 45.246353 & -7.1 \\
\hline ZINC33900348 & $\begin{array}{l}6954466,6746658,16411215, \\
5419445\end{array}$ & 41.02396 & 44.132156 & $\begin{array}{l}-7.1 \\
1\end{array}$ \\
\hline ZINC67851876 & 56754785 & 41.18465 & 45.03601 & -7.1 \\
\hline ZINC06621638 & 4594961 & 41.158195 & 44.026863 & -7.1 \\
\hline ZINC72237750 & 66488018 & 41.000103 & 43.6998 & -7.1 \\
\hline ZINC67587504 & 56703644 & 40.40443 & 44.093094 & -7.1 \\
\hline ZINC11568533 & 24253070,42035190 & 41.394897 & 44.75829 & -7.1 \\
\hline ZINC33171252 & 46394772 & 41.106136 & 44.558796 & -7.1 \\
\hline ZINC13735083 & 44378620 & 39.85497 & 45.85691 & -7.1 \\
\hline ZINC22151060 & 30055454,16778495 & 40.076927 & 44.980507 & -7.1 \\
\hline ZINC01703954 & $419266,24190704,54606148$ & 39.758102 & 45.28904 & -7.1 \\
\hline ZINC69743339 & 53601970 & 41.147705 & 44.639008 & -7.1 \\
\hline
\end{tabular}




\begin{tabular}{|c|c|c|c|c|}
\hline ZINC03073295 & 2303199 & 41.14471 & 44.7706 & -7.1 \\
\hline ZINC12745511 & 45204349,25478001 & 41.03456 & 43.32128 & -7.1 \\
\hline ZINC43153676 & 24856343 & 40.112053 & 43.268696 & -7.1 \\
\hline ZINC06118685 & 10426130,152936 & 40.04765 & 44.630592 & -7.1 \\
\hline ZINC78601482 & 60289429 & 40.127502 & 44.048016 & -7.1 \\
\hline ZINC44126642 & 59060916 & 39.741856 & 43.914867 & -7.1 \\
\hline ZINC04648807 & $6526289,6091695,6826544$ & 41.315254 & 45.583435 & -7.1 \\
\hline ZINC07259358 & 51429980,8360585 & 40.915203 & 44.00082 & -7.1 \\
\hline ZINC35479578 & 45509468 & 39.67714 & 43.531384 & -7.1 \\
\hline ZINC57509546 & 52454716,49814399 & 41.455032 & 43.146233 & -7.1 \\
\hline ZINC20529000 & 704368,6924055 & 39.651836 & 45.46312 & -7.1 \\
\hline ZINC35325003 & 42648017 & 41.314438 & 43.91197 & -7.1 \\
\hline ZINC17124519 & $51571345,1361573,2890201$ & 40.30331 & 43.499435 & -7.1 \\
\hline ZINC38008845 & 61004238 & 40.011734 & 43.124363 & -7.1 \\
\hline ZINC19427820 & 24691436,28173940 & 40.18515 & 46.130672 & -7.1 \\
\hline ZINC04023622 & 57381417 & 40.188927 & 44.116333 & -7.1 \\
\hline ZINC05343446 & 5624268,6776438 & 41.162 & 44.598103 & -7.1 \\
\hline ZINC00379353 & $\begin{array}{l}\text { 6779455,846527,53460051, } \\
2885748\end{array}$ & 41.209892 & 43.523678 & -7.1 \\
\hline ZINC00379354 & \begin{tabular}{|l|}
$846528,2885748,53460051$, \\
6949650 \\
\end{tabular} & 41.215874 & 43.837772 & -7.1 \\
\hline ZINC00353986 & 6948426,830856 & 40.570007 & 45.73753 & -7.1 \\
\hline ZINC20478480 & 28977009 & 39.5567 & 43.975143 & -7.1 \\
\hline ZINC35463145 & 50744087 & \begin{tabular}{|l|l|}
39.674706 \\
\end{tabular} & 42.870583 & -7.1 \\
\hline ZINC71747084 & 56869741 & 39.657223 & 45.358036 & -7.1 \\
\hline ZINC20119698 & 28642269,45233059 & 41.12761 & 44.495285 & -7.1 \\
\hline ZINC36621394 & 51856811,45925254 & 40.919262 & 44.43344 & -7.1 \\
\hline ZINC40542903 & 45828839 & 41.163986 & 44.150272 & -7.1 \\
\hline ZINC71890769 & 56785803 & 41.243805 & 43.87778 & -7.1 \\
\hline ZINC08973017 & 5614187,6775377 & 40.83102 & 44.42805 & -7.1 \\
\hline ZINC08329230 & 9454244,16304771 & 40.37354 & 44.02533 & -7.1 \\
\hline ZINC08040502 & 9172364 & 40.018234 & 46.12418 & -7.1 \\
\hline ZINC04893856 & 773430,7405954 & 41.41952 & 44.54358 & -7.1 \\
\hline ZINC37593687 & 45356734 & 41.092766 & 45.769062 & -7.1 \\
\hline ZINC67769971 & 56708428 & 39.835106 & 43.57866 & -7.1 \\
\hline ZINC02306438 & 1924583 & 40.023003 & 45.617058 & -7.1 \\
\hline ZINC28807290 & 35971096 & 41.008244 & 43.96681 & -7.1 \\
\hline ZINC65395918 & 50959887 & 40.18491 & 45.48304 & -7.1 \\
\hline ZINC04002943 & 1472839,40470071 & 40.968307 & 43.485027 & -7.1 \\
\hline ZINC10814751 & 41677010 & 41.236656 & 43.2523 & -7.1 \\
\hline ZINC31990661 & 39218484 & 39.856876 & 43.027485 & -7.1 \\
\hline ZINC13212186 & $6798781,44723041,5384963$ & 39.83423 & 43.786835 & -7.1 \\
\hline ZINC04367037 & 3328890,9620057 & 40.009735 & 43.35966 & -7.1 \\
\hline ZINC02479297 & 2055430 & 41.48675 & 44.852623 & -7.1 \\
\hline ZINC75251488 & 56688577 & 41.62452 & 43.59221 & -7.1 \\
\hline ZINC34150018 & 10062341 & 40.440777 & 43.80917 & -7.1 \\
\hline ZINC12560330 & 25358656 & 39.768482 & 42.59155 & -7.1 \\
\hline ZINC36559166 & 51802202,45490311 & 41.032528 & 43.034027 & -7.1 \\
\hline ZINC08394171 & 9507921,16305469 & 41.161377 & 43.40175 & -7.1 \\
\hline ZINC04763921 & 5306165,7350526 & 41.252296 & 45.823727 & -7.1 \\
\hline ZINC49452907 & 53212200 & 40.174294 & 44.410755 & -7.1 \\
\hline ZINC11802242 & 24271345,42181872 & 40.04528 & 45.0937 & -7.1 \\
\hline ZINC11802244 & 24271345,42181874 & 40.047657 & 45.09449 & -7.1 \\
\hline ZINC13534362 & 25794349,18524185 & 40.67708 & 43.431587 & -7.1 \\
\hline ZINC30519629 & 37623775 & 40.997864 & 45.15601 & -7.1 \\
\hline ZINC51325933 & 52339346,46859034 & 41.48996 & 43.953396 & -7.1 \\
\hline ZINC49466311 & 53214344 & 41.348255 & 43.37041 & -7.1 \\
\hline
\end{tabular}




\begin{tabular}{|c|c|c|c|c|}
\hline ZINC49031938 & 52314557,49670000 & 41.25287 & 43.175472 & -7.1 \\
\hline ZINC12594138 & 45195871,25382833 & 41.033466 & 43.304306 & -7.1 \\
\hline ZINC12145983 & 46959745 & 41.391193 & 44.58183 & -7.1 \\
\hline ZINC04655851 & $1207459,1776089,7002495$ & 40.679493 & 45.576653 & -7.1 \\
\hline ZINC13221114 & 367586 & 39.653896 & 45.24173 & -7.1 \\
\hline ZINC44870226 & 52207394,45796266 & 39.959576 & 44.037086 & -7.1 \\
\hline ZINC05336934 & 40527499,14767281 & 39.5366 & 44.21649 & -7.1 \\
\hline ZINC05221531 & 7564243,16301638 & 41.1497 & 44.36785 & -7.1 \\
\hline ZINC16778625 & 27331693,16443333 & 40.001617 & 44.079422 & -7 \\
\hline ZINC13004762 & 25663844 & 40.860195 & 44.439022 & -7 \\
\hline ZINC69778079 & 53605417 & 40.932888 & 44.71168 & -7 \\
\hline ZINC65415696 & 53503146 & 40.38713 & 43.612904 & -7 \\
\hline ZINC33559806 & $\begin{array}{l}6747207,4601096,7461055, \\
4916496,53351474,6348654\end{array}$ & 40.005264 & 43.236637 & -7 \\
\hline ZINC25804238 & 33078347,45801776 & 41.447304 & 44.35677 & -7 \\
\hline ZINC19915386 & 28452067 & 40.575356 & 44.43803 & -7 \\
\hline ZINC21727926 & 46374870 & 40.942135 & 43.30319 & -7 \\
\hline ZINC71905431 & 56790246 & 40.21015 & 44.16057 & -7 \\
\hline ZINC29019930 & 36193956 & 40.991497 & 43.330673 & -7 \\
\hline ZINC71405224 & 53469875 & 40.80446 & 45.964596 & -7 \\
\hline ZINC12531697 & 17450908 & 40.99653 & 44.307064 & -7 \\
\hline ZINC05796428 & 7802040,16312622 & 40.2172 & 44.13439 & -7 \\
\hline ZINC25761401 & 5091694,9607380 & 41.034973 & 43.49418 & -7 \\
\hline ZINC71328557 & 53367371 & 40.99476 & 45.3841 & -7 \\
\hline ZINC57024346 & $52418412,17438271,40790814$ & 40.072227 & 43.545452 & -7 \\
\hline ZINC26508196 & 33779363,43841820 & 41.19757 & 43.232346 & -7 \\
\hline ZINC35575798 & 4737582 & 40.686867 & 43.994835 & -7 \\
\hline ZINC05494681 & 5001297,9688250 & 41.035393 & 43.49519 & -7 \\
\hline ZINC09228234 & 24793726 & 40.98093 & 44.650513 & -7 \\
\hline ZINC69483915 & 53569047 & 40.895226 & 44.856678 & -7 \\
\hline ZINC06345388 & 4203238 & 40.213165 & 45.60454 & -7 \\
\hline ZINC58297510 & 51087505 & 41.100998 & 43.81546 & -7 \\
\hline ZINC12443244 & 25283436 & 40.496655 & 44.90116 & -7 \\
\hline ZINC06050195 & 4066014 & 41.21646 & 45.30012 & -7 \\
\hline ZINC31861706 & 39079146 & 39.506344 & 43.96098 & -7 \\
\hline ZINC31861859 & 39079213 & 39.505024 & 43.962555 & -7 \\
\hline ZINC58180419 & 47076108 & 41.1077 & 43.276123 & -7 \\
\hline ZINC28402558 & 5161759,24210872 & 40.96769 & 45.4841 & -7 \\
\hline ZINC67515052 & 56702831 & 41.21131 & 43.14592 & -7 \\
\hline ZINC71721674 & 56867414 & 40.554184 & 45.817013 & -7 \\
\hline ZINC55107951 & 52382418,46985864 & 41.327003 & 45.642326 & -7 \\
\hline ZINC20327501 & 977093 & 40.431335 & 43.53682 & -7 \\
\hline ZINC20356047 & 43985675 & 39.989975 & 44.749977 & -7 \\
\hline ZINC02237742 & $5521906,51383569,6793039$ & 39.998188 & 44.006447 & -7 \\
\hline ZINC19523981 & 46972964 & 40.16474 & 44.20465 & -7 \\
\hline ZINC49452891 & 53212186 & 40.216312 & 44.43285 & -7 \\
\hline ZINC72192423 & 66520553 & 40.97426 & 43.481586 & -7 \\
\hline ZINC19722956 & 28287485 & 41.123886 & 45.720093 & -7 \\
\hline ZINC40168659 & 43828475 & 39.908737 & 43.102676 & -7 \\
\hline ZINC72170208 & 56915800 & 41.296265 & 44.356018 & -7 \\
\hline ZINC12561116 & $51510140,2264664,2881425$ & 39.925415 & 44.233204 & -7 \\
\hline ZINC71921950 & 56795377 & 40.938404 & 43.56423 & -7 \\
\hline ZINC08133667 & 9268686 & 40.03415 & 43.881012 & -7 \\
\hline ZINC71858301 & 60425235 & 40.934647 & 44.364292 & -7 \\
\hline ZINC24903169 & 24590234 & 41.29679 & 44.358738 & -7 \\
\hline ZINC03191756 & 44784324,4561576 & 39.955452 & 44.98633 & -7 \\
\hline ZINC61966031 & 52903843 & 41.113705 & 43.086063 & -7 \\
\hline
\end{tabular}




\begin{tabular}{|c|c|c|c|c|}
\hline ZINC45947088 & 21833467 & 41.168568 & 43.677326 & -7 \\
\hline ZINC20313503 & 46243543 & 39.790543 & 44.974003 & -7 \\
\hline ZINC69438383 & 53563125 & 40.895462 & 44.85344 & -7 \\
\hline ZINC09304366 & 41009109,16415810 & 40.356667 & 44.59646 & -7 \\
\hline ZINC21531745 & 46281824 & 40.937637 & 43.302162 & -7 \\
\hline ZINC07875634 & 53500137,8996426 & 40.882484 & 45.60493 & -7 \\
\hline ZINC09109980 & $\begin{array}{l}7695087,40937384,4544232,51453 \\
502\end{array}$ & 40.262714 & 43.940193 & -7 \\
\hline ZINC09109981 & $\begin{array}{l}18556169,40937385,51453503,454 \\
4232,7695090\end{array}$ & 40.262535 & 43.611908 & -7 \\
\hline ZINC31907835 & 39118969 & 39.505333 & 44.498573 & -7 \\
\hline ZINC71890770 & 56785803 & 41.2442 & 42.85553 & -7 \\
\hline ZINC03289292 & 40459659,2333499 & 40.939964 & 43.274246 & -7 \\
\hline ZINC67867938 & 56717938 & 39.8574 & 43.31916 & -7 \\
\hline ZINC71890767 & 56785803 & 41.243805 & 43.87778 & -7 \\
\hline ZINC09134773 & 40947377,16426348 & 40.356647 & 45.30016 & -7 \\
\hline ZINC46494407 & 56796159 & 41.4467 & 44.657337 & -7 \\
\hline ZINC25874961 & 33146991 & 40.98767 & 44.84031 & -7 \\
\hline ZINC64987250 & 53097503 & 40.886528 & 44.883835 & -7 \\
\hline ZINC34973547 & 43150954 & 40.0127 & 45.2931 & -7 \\
\hline ZINC58956298 & 53614097 & 41.153458 & 42.991776 & -7 \\
\hline ZINC21114636 & 22585566 & 40.991257 & 43.122257 & -7 \\
\hline ZINC06451480 & 6415576,6827368 & 40.042656 & 43.56183 & -7 \\
\hline ZINC55392846 & 52399247,46998180 & 41.09161 & 44.32345 & -7 \\
\hline ZINC19790508 & $\begin{array}{l}3303636,675304,6920059, \\
3296004,3129060\end{array}$ & 39.868942 & 44.272404 & -7 \\
\hline ZINC20325320 & 976365 & 40.354004 & 43.581367 & -7 \\
\hline ZINC72192406 & 66520434 & 41.214314 & 44.25156 & -7 \\
\hline ZINC53555427 & 47057913 & 40.2172 & 44.903996 & -7 \\
\hline ZINC25464072 & 51648635,24654119 & 40.884026 & 45.603714 & -7 \\
\hline ZINC58343644 & 51108533 & 39.780228 & 45.271885 & -7 \\
\hline ZINC20472820 & $\begin{array}{l}16432720,54706664,5413089, \\
6744813,6951675\end{array}$ & 40.36066 & 45.189804 & -7 \\
\hline ZINC55175262 & 51063590 & 41.030975 & 43.54723 & -7 \\
\hline ZINC38486357 & 51893853,45498065 & 41.11357 & 43.17528 & -7 \\
\hline ZINC08230710 & 9361259 & 41.190872 & 42.599407 & -7 \\
\hline ZINC06188072 & 6825465,18528135 & 40.384216 & 42.99287 & -6.9 \\
\hline ZINC13940876 & 3659443 & 39.885746 & 43.579113 & -6.9 \\
\hline ZINC01367299 & 1459497,3216795 & 41.226242 & 44.537384 & -6.9 \\
\hline ZINC71806341 & 60242216 & 41.114544 & 43.231308 & -6.9 \\
\hline ZINC55154202 & 46987653 & 41.149036 & 44.635796 & -6.9 \\
\hline ZINC36614300 & 44822093 & 41.095757 & 42.59804 & -6.9 \\
\hline ZINC04741868 & $7342907,16473742,28450897$ & 40.282707 & 44.35299 & -6.9 \\
\hline ZINC32921986 & 40159502 & 40.05221 & 43.259186 & -6.9 \\
\hline ZINC01282250 & 44162895,1405064 & 40.77806 & 43.267 & -6.9 \\
\hline ZINC23134834 & 30858318,42204389 & 40.702705 & 45.308502 & -6.9 \\
\hline ZINC04763918 & 5306165,7350524 & 41.25048 & 45.4367 & -6.9 \\
\hline ZINC12504722 & $25323144,3790389,2117387$ & 39.552425 & 44.437775 & -6.9 \\
\hline ZINC09726413 & 17409069 & 40.099545 & 43.858765 & -6.9 \\
\hline ZINC38740336 & 45509513 & 39.675808 & 44.66094 & -6.9 \\
\hline ZINC68297159 & 60230839 & 41.018604 & 43.10053 & -6.9 \\
\hline ZINC03225009 & 2343193 & 40.162556 & 45.064835 & -6.9 \\
\hline ZINC15130755 & 60412955 & 40.315384 & 44.969776 & -6.9 \\
\hline ZINC38933264 & 43972173 & 41.083553 & 44.271748 & -6.9 \\
\hline ZINC55245404 & 52390378,46991488 & 40.98537 & 44.54139 & -6.9 \\
\hline ZINC71285467 & 60181486 & 40.51029 & 43.42007 & -6.9 \\
\hline ZINC20356048 & 43985675 & 39.989975 & 44.685356 & -6.9 \\
\hline
\end{tabular}




\begin{tabular}{|c|c|c|c|c|}
\hline ZINC28382069 & 24211589,35655317 & 40.987816 & 44.80889 & -6.9 \\
\hline ZINC72169200 & 56914860 & 41.02098 & 44.22751 & -6.9 \\
\hline ZINC66814555 & 53626141 & 40.644493 & 43.2339 & -6.9 \\
\hline ZINC67472885 & 50950432 & 40.891605 & 43.758617 & -6.9 \\
\hline ZINC45471692 & 46615753 & 41.169025 & 42.476616 & -6.9 \\
\hline ZINC20119700 & 28642271,45233059 & 41.1275 & 44.631313 & -6.9 \\
\hline ZINC71962722 & 60519188 & 40.963596 & 43.946037 & -6.9 \\
\hline ZINC05958183 & 5417973 & 40.952145 & 44.089237 & -6.9 \\
\hline ZINC06752115 & 16011713 & 41.31571 & 44.23715 & -6.9 \\
\hline ZINC00000654 & $\begin{array}{l}\text { 23687372,69612922,9848893, } \\
21568497,67938695,4888, \\
70362942,9929564,67597037, \\
\text { 42606169,70273661,9936064, } \\
25271719,70270364\end{array}$ & 40.67917 & 44.65943 & -6.9 \\
\hline ZINC72420480 & 56891943 & 40.739525 & 46.042446 & -6.9 \\
\hline ZINC13526387 & $44350836,49796651,10935302$ & 40.190834 & 44.784134 & -6.9 \\
\hline ZINC15444270 & 51546302,26598898 & 41.201485 & 44.161465 & -6.9 \\
\hline ZINC04623170 & 54694294 & 40.36579 & 43.165977 & -6.9 \\
\hline ZINC67730166 & 40523418,3743379 & 39.887474 & 43.312584 & -6.9 \\
\hline ZINC13726358 & 51527013,17601049 & 40.979984 & 44.051414 & -6.9 \\
\hline ZINC12594141 & 45195871,25382836 & 41.034126 & 44.41262 & -6.9 \\
\hline ZINC49453627 & 53212750 & 41.0018 & 44.80889 & -6.9 \\
\hline ZINC49453626 & 53212750 & 41.0018 & 45.321793 & -6.9 \\
\hline ZINC05517121 & 3848269,40541898 & 41.017265 & 44.45123 & -6.9 \\
\hline ZINC11372867 & 41947638 & 41.159172 & 43.409252 & -6.9 \\
\hline ZINC71958247 & 60514808 & 41.131344 & 42.940876 & -6.9 \\
\hline ZINC35672562 & 51699449,46300367 & 41.03831 & 43.28078 & -6.9 \\
\hline ZINC73634496 & 60181606 & 40.82872 & 45.86289 & -6.9 \\
\hline ZINC16940894 & 21787551,5239076 & 41.20169 & 44.011845 & -6.9 \\
\hline ZINC57783910 & 60230909 & 41.09298 & 43.57758 & -6.9 \\
\hline ZINC69588448 & 53584092 & 40.961754 & 44.054867 & -6.9 \\
\hline ZINC41378157 & 53532662,52142759 & 40.995434 & 43.875927 & -6.9 \\
\hline ZINC19946319 & 28470780 & 39.562485 & 45.018898 & -6.9 \\
\hline ZINC04187936 & 51400676,3173102 & 40.256443 & 45.03568 & -6.9 \\
\hline ZINC12793866 & 24237814 & 41.390648 & 44.28444 & -6.9 \\
\hline ZINC12812153 & 42917021,51513401 & 39.77055 & 43.881363 & -6.9 \\
\hline ZINC71753023 & 56873735 & 40.995697 & 45.075462 & -6.9 \\
\hline ZINC71767528 & 56880008 & 40.260616 & 44.4217 & -6.9 \\
\hline ZINC16449130 & $18530425,27137323,23937530$ & 41.46445 & 43.386894 & -6.9 \\
\hline ZINC02234356 & 1416244,5520481 & 41.08884 & 44.342545 & -6.9 \\
\hline ZINC28382001 & 24211556,35655263 & 40.988663 & 44.808273 & -6.9 \\
\hline ZINC04305921 & 7179591,4258779 & 39.94005 & 43.739742 & -6.9 \\
\hline ZINC05374648 & $7657215,4762770,6207152$ & 40.236546 & 45.35854 & -6.9 \\
\hline ZINC16513306 & $5332960,2223529,6743338$ & 40.197865 & 44.01899 & -6.9 \\
\hline ZINC04656182 & 7311192,919191 & 39.224014 & 42.880608 & -6.9 \\
\hline ZINC46494403 & 56796159 & 41.447964 & 44.65566 & -6.9 \\
\hline ZINC31648442 & 38869401,47006626 & 40.979385 & 44.019875 & -6.9 \\
\hline ZINC03872070 & $\begin{array}{l}\text { 68648461,11562090,56676308, } \\
25200543,69450862,20431397, \\
21814519,66762077,68816707, \\
5281607,70129138\end{array}$ & 39.706787 & 43.282345 & -6.9 \\
\hline ZINC30520532 & 37625059 & 40.779278 & 45.4106 & -6.9 \\
\hline ZINC01270130 & $34364612,933411,5421930$ & 41.05561 & 44.378067 & -6.9 \\
\hline ZINC27551100 & 6826800 & 40.214516 & 45.641975 & -6.9 \\
\hline ZINC07875632 & 53500137,8996424 & 40.882484 & 45.60493 & -6.9 \\
\hline ZINC05025258 & $\begin{array}{l}\text { 4601096,44660832,16682482, } \\
52994460,9613806,6747207,\end{array}$ & 40.00376 & 43.642273 & -6.9 \\
\hline
\end{tabular}




\begin{tabular}{|c|c|c|c|c|}
\hline & 4916496,53351474 & & & \\
\hline ZINC06922328 & 4661590 & 39.113163 & 45.123337 & -6.9 \\
\hline ZINC04635051 & 7307696,716071 & 40.965656 & 43.441566 & -6.9 \\
\hline ZINC22835259 & 24527894 & 41.391674 & 44.848804 & -6.9 \\
\hline ZINC39947495 & 51921981,47002247 & 40.26637 & 45.17055 & -6.9 \\
\hline ZINC19689698 & 28283521 & 41.15668 & 44.862354 & -6.9 \\
\hline ZINC20177606 & 28702575,5135207 & 40.41065 & 43.929886 & -6.9 \\
\hline ZINC15673774 & 26743331 & 40.964962 & 44.974552 & -6.9 \\
\hline ZINC33702524 & $3591444,25829648,5869482$ & 40.240112 & 43.504864 & -6.8 \\
\hline ZINC01367298 & 1459496,3216795 & 41.22472 & 44.998924 & -6.8 \\
\hline ZINC72423393 & 56884611 & 40.930885 & 43.400635 & -6.8 \\
\hline ZINC14140762 & 17453252,25959989 & 41.123737 & 44.669056 & -6.8 \\
\hline ZINC11757366 & 22693643,42150076 & 40.1373 & 44.887146 & -6.8 \\
\hline ZINC19109522 & 24420180 & 39.589027 & 44.168514 & -6.8 \\
\hline ZINC28965248 & 36132716 & 40.804386 & 43.656105 & -6.8 \\
\hline ZINC20357853 & 28875646 & 41.21825 & 44.4913 & -6.8 \\
\hline ZINC00210508 & 755657 & 40.501625 & 43.43149 & -6.8 \\
\hline ZINC67872741 & 56711690 & 39.834007 & 42.919823 & -6.8 \\
\hline ZINC71958221 & 60514527 & 41.245094 & 44.559765 & -6.8 \\
\hline ZINC37674847 & 60869914 & 40.837 & 44.047867 & -6.8 \\
\hline ZINC32699245 & $3353645,39929799,5801681$ & 40.193317 & 44.53439 & -6.8 \\
\hline ZINC67172463 & 52940301 & 40.988914 & 42.85911 & -6.8 \\
\hline ZINC71856761 & 56801837 & 40.97167 & 44.00421 & -6.8 \\
\hline ZINC05516954 & 40541878,3813177 & 41.13404 & 45.89329 & -6.8 \\
\hline ZINC31737205 & 42952317,38959853 & 41.10206 & 43.646152 & -6.8 \\
\hline ZINC04451128 & 3156299 & 41.12571 & 44.80259 & -6.8 \\
\hline ZINC31737202 & 42952317,38959850 & 41.094284 & 44.290546 & -6.8 \\
\hline ZINC05494688 & 5152900,9609774 & 41.031967 & 43.84059 & -6.8 \\
\hline ZINC13125417 & 51517433 & 41.175076 & 42.62426 & -6.8 \\
\hline ZINC66738334 & 53612698 & 41.233383 & 44.369347 & -6.8 \\
\hline ZINC50603829 & 49382725 & 40.18778 & 43.460873 & -6.8 \\
\hline ZINC71285042 & 53370830 & 40.510185 & 43.28447 & -6.8 \\
\hline ZINC35672559 & 51699447,46300367 & 41.03831 & 43.28078 & -6.8 \\
\hline ZINC06438299 & 5665177 & 40.587864 & 43.606693 & -6.8 \\
\hline ZINC65575656 & 53547626 & 39.912407 & 44.436256 & -6.8 \\
\hline ZINC00269966 & $3149492,778287,6942351$ & 40.420673 & 44.692287 & -6.8 \\
\hline ZINC26710628 & 33993674 & 40.299294 & 43.768585 & -6.8 \\
\hline ZINC02518806 & 179635 & 40.20199 & 45.08964 & -6.8 \\
\hline ZINC07652746 & 51431166,4789830 & 41.213356 & 42.669765 & -6.8 \\
\hline ZINC08981253 & 17557948 & 39.508347 & 44.273964 & -6.8 \\
\hline ZINC44913254 & 52210034,45813011 & 40.866898 & 44.592884 & -6.8 \\
\hline ZINC40478836 & 51981598,45030991 & 40.743538 & 45.570847 & -6.8 \\
\hline ZINC20737138 & 29136372,42652492 & 41.312405 & 45.509705 & -6.8 \\
\hline ZINC44978444 & 45834540 & 40.966846 & 45.273705 & -6.8 \\
\hline ZINC02508405 & 40423596,5102083 & 40.654316 & 43.637135 & -6.8 \\
\hline ZINC49454484 & 53213459 & 40.707054 & 44.341595 & -6.8 \\
\hline ZINC20489238 & 22521107 & 40.95907 & 44.50521 & -6.8 \\
\hline ZINC55283522 & 52393123,50970826 & 41.23823 & 43.558777 & -6.8 \\
\hline ZINC03043400 & 4064269,9644911 & 41.245216 & 43.659252 & -6.8 \\
\hline ZINC68116874 & 48186623 & 40.821945 & 43.60999 & -6.8 \\
\hline ZINC25464032 & 24654098,32768044 & 41.391743 & 43.160927 & -6.8 \\
\hline ZINC71910954 & 56792396 & 41.094242 & 45.925106 & -6.8 \\
\hline ZINC35320770 & 43445097 & 41.013912 & 44.97262 & -6.8 \\
\hline ZINC23107046 & 30835302,42952393 & 41.39113 & 44.58579 & -6.8 \\
\hline ZINC49453932 & 53212990 & 40.199394 & 45.7024 & -6.8 \\
\hline ZINC05332550 & 3797678 & 40.019535 & 44.410465 & -6.8 \\
\hline ZINC03850817 & 40467702,2346869 & 40.240696 & 44.37606 & -6.8 \\
\hline
\end{tabular}




\begin{tabular}{|c|c|c|c|c|}
\hline ZINC13119596 & 6412980,6761350 & 39.961884 & 44.077057 & -6.8 \\
\hline ZINC71914885 & 56793666 & 41.042274 & 45.154583 & -6.8 \\
\hline ZINC18192156 & $1256183,2978361,51579671$ & 41.09184 & 43.461548 & -6.8 \\
\hline ZINC35669012 & 4771079 & 41.13005 & 45.34586 & -6.8 \\
\hline ZINC37296615 & 54729628,43318313 & 40.99231 & 44.012497 & -6.8 \\
\hline ZINC03418768 & 51396092,2537940 & 40.658554 & 43.601425 & -6.8 \\
\hline ZINC12902251 & 25589579,42888007 & 41.39199 & 44.490776 & -6.8 \\
\hline ZINC35021941 & 44782736 & 40.465706 & 44.563763 & -6.8 \\
\hline ZINC20313507 & 46243543 & 39.79092 & 45.017235 & -6.8 \\
\hline ZINC07010589 & 40660633,4728735 & 40.169262 & 44.52064 & -6.8 \\
\hline ZINC71890768 & 56785803 & 41.2442 & 44.253407 & -6.8 \\
\hline ZINC02479470 & 2931238,51386462 & 40.19468 & 43.627556 & -6.8 \\
\hline ZINC72236803 & 66487047 & 41.091877 & 44.795498 & -6.8 \\
\hline ZINC33818908 & 5278575 & 40.06478 & 44.723614 & -6.8 \\
\hline ZINC13427803 & 45342473 & 40.015884 & 45.31727 & -6.8 \\
\hline ZINC00612664 & $3304547,2958945,972448,6594270$ & 41.14985 & 45.105404 & -6.8 \\
\hline ZINC03831354 & $\begin{array}{l}23687372,69612922,9929564, \\
21568497,67938695,4888, \\
70362942,9936064,67597037, \\
42606169,70273661,40467221, \\
9848893,70270364\end{array}$ & 40.680004 & 43.977333 & -6.8 \\
\hline ZINC27788982 & 35177227 & 41.006218 & 44.70461 & -6.8 \\
\hline ZINC05675790 & $\begin{array}{l}\text { 7189018,40554559,2808527, } \\
5899217\end{array}$ & 39.86341 & 45.207043 & -6.8 \\
\hline ZINC34926759 & 51674356,25220245 & 40.980667 & 43.00547 & -6.8 \\
\hline ZINC29194460 & 36380873 & 40.41377 & 42.97255 & -6.8 \\
\hline ZINC12561276 & 25359112 & 40.84542 & 44.878014 & -6.8 \\
\hline ZINC16778467 & 4907485,27331577 & 39.999706 & 46.074116 & -6.8 \\
\hline ZINC69566563 & 53580800 & 41.04631 & 43.817802 & -6.8 \\
\hline ZINC13690067 & 17596164,25846746 & 39.522762 & 44.346027 & -6.8 \\
\hline ZINC05750122 & 1481309,40561051 & 41.001198 & 44.45462 & -6.8 \\
\hline ZINC41194273 & 52985684,52113608 & 41.144146 & 43.30159 & -6.8 \\
\hline ZINC19791126 & 45225185,28357604 & 40.293686 & 45.71059 & -6.8 \\
\hline ZINC49949610 & 52330817 & 40.59816 & 44.673767 & -6.8 \\
\hline ZINC00432767 & 5336331,867976 & 41.149857 & 43.657887 & -6.8 \\
\hline ZINC08585017 & $\begin{array}{l}\text { 44134842,3272523,67426225, } \\
20835491,6098006,44281561, \\
\text { 10134355,67326670,9895610, } \\
25162983,4577404\end{array}$ & 41.115242 & 43.772797 & -6.8 \\
\hline ZINC01270131 & $6859144,933411,51379590$ & 41.05749 & 44.149902 & -6.8 \\
\hline ZINC05007280 & 3141872,9594993 & 41.035397 & 43.84244 & -6.8 \\
\hline ZINC03156109 & 4675052 & 40.595848 & 45.73218 & -6.8 \\
\hline ZINC05221540 & 7564235,16301638 & 41.15088 & 44.233486 & -6.8 \\
\hline ZINC16778452 & 4905185,27331567 & 40.003204 & 46.074013 & -6.8 \\
\hline ZINC09344715 & 51458811,16426210 & 40.813747 & 44.46672 & -6.8 \\
\hline ZINC21954031 & 29893940,16770671 & 40.642952 & 45.755196 & -6.7 \\
\hline ZINC35843254 & $3104949,7322934,54736111$ & 40.113007 & 43.990788 & -6.7 \\
\hline ZINC21782083 & 44121644 & 40.4755 & 45.68029 & -6.7 \\
\hline ZINC71851858 & 56800659 & 41.140083 & 43.23829 & -6.7 \\
\hline ZINC25464037 & 24654098,32768049 & 41.391808 & 43.278954 & -6.7 \\
\hline ZINC72402883 & 60180772 & 39.813084 & 44.067486 & -6.7 \\
\hline ZINC05010604 & 7454181,16461774 & 40.011253 & 42.655434 & -6.7 \\
\hline ZINC71405405 & 56766456 & 40.21015 & 43.505753 & -6.7 \\
\hline ZINC69740856 & 53601222 & 40.209244 & 43.58589 & -6.7 \\
\hline ZINC40177521 & 46398601 & 40.38644 & 44.47969 & -6.7 \\
\hline ZINC00269850 & 3493348 & 40.384804 & 44.288326 & -6.7 \\
\hline ZINC13470536 & 51522369 & 41.02129 & 43.265583 & -6.7 \\
\hline
\end{tabular}




\begin{tabular}{|c|c|c|c|c|}
\hline ZINC32265857 & 620273 & 41.474262 & 43.30316 & -6.7 \\
\hline ZINC23644726 & 31282659 & 39.977512 & 43.587936 & -6.7 \\
\hline ZINC66738336 & 53612698 & 41.233845 & 44.38163 & -6.7 \\
\hline ZINC03433477 & 2553090 & 41.26394 & 43.55411 & -6.7 \\
\hline ZINC19724010 & 28288815 & 41.427643 & 44.687294 & -6.7 \\
\hline ZINC65371881 & 50949880 & 39.736008 & 45.503166 & -6.7 \\
\hline ZINC12902245 & 25589575,42888007 & 41.391144 & 43.5282 & -6.7 \\
\hline ZINC71759311 & 56876119 & 40.042076 & 44.52329 & -6.7 \\
\hline ZINC12745516 & 45204349,25478007 & 41.03363 & 43.245583 & -6.7 \\
\hline ZINC04187941 & $861762,40478067,3173102$ & 40.257866 & 44.637756 & -6.7 \\
\hline ZINC09644460 & 51466718,16352693 & 41.27789 & 44.295547 & -6.7 \\
\hline ZINC04050369 & 2767913,9581905 & 41.188675 & 43.644882 & -6.7 \\
\hline ZINC71285468 & 60181486 & 40.50986 & 45.3751 & -6.7 \\
\hline ZINC05225034 & 7567137 & 41.136265 & 44.77643 & -6.7 \\
\hline ZINC72474807 & 56911228 & 41.377785 & 43.437416 & -6.7 \\
\hline ZINC00133571 & 5397925 & 40.23297 & 42.71378 & -6.7 \\
\hline ZINC00398864 & 4253048 & 40.135677 & 43.99149 & -6.7 \\
\hline ZINC69566412 & 53580763 & 40.409737 & 44.545013 & -6.7 \\
\hline ZINC65515593 & 53510488 & 41.100525 & 44.126152 & -6.7 \\
\hline ZINC24901680 & 32211393 & 40.897522 & 43.172554 & -6.7 \\
\hline ZINC71904401 & 56789755 & 40.896152 & 45.306286 & -6.7 \\
\hline ZINC22147510 & 6732999,5347293 & 40.468136 & 44.361298 & -6.7 \\
\hline ZINC00108487 & 11878614,714010 & 40.6318 & 45.23228 & -6.7 \\
\hline ZINC71285511 & 56762052 & 40.122044 & 43.663464 & -6.7 \\
\hline ZINC72429421 & 56885079 & 41.03531 & 44.07579 & -6.7 \\
\hline ZINC65434227 & 50971493 & 40.150494 & 44.755493 & -6.7 \\
\hline ZINC13140286 & 51518064 & 40.137398 & 44.04275 & -6.7 \\
\hline ZINC08723783 & 17431758,40797142 & 39.877014 & 44.486023 & -6.7 \\
\hline ZINC17734755 & 24528332 & 39.92295 & 44.911976 & -6.7 \\
\hline ZINC00133814 & 6928278,726076 & 41.21808 & 44.25497 & -6.7 \\
\hline ZINC29004357 & 42930488,36177130 & 40.08895 & 43.42101 & -6.7 \\
\hline ZINC44977992 & 45834387 & 41.14672 & 45.695793 & -6.7 \\
\hline ZINC71893935 & 56786442 & 41.148373 & 45.00422 & -6.7 \\
\hline ZINC19581130 & 28229558 & 41.004032 & 44.462765 & -6.7 \\
\hline ZINC12505442 & 25323640,16227883 & 41.15836 & 44.34797 & -6.7 \\
\hline ZINC71747085 & 56869741 & 39.662556 & 45.15425 & -6.7 \\
\hline ZINC04023653 & 40471096,1479314 & 41.091316 & 43.291 & -6.7 \\
\hline ZINC16690018 & 18575851 & 39.79191 & 44.81558 & -6.7 \\
\hline ZINC71335711 & 53371341 & 40.99788 & 45.84258 & -6.7 \\
\hline ZINC02860465 & $2213624,3304547,2958945$ & 41.14999 & 45.156143 & -6.7 \\
\hline ZINC41194276 & 52985684,52113610 & 41.145046 & 43.30193 & -6.7 \\
\hline ZINC33372316 & $5707721,40472010,2767913$ & 41.269253 & 44.01043 & -6.7 \\
\hline ZINC01648143 & $\begin{array}{l}24184018,4587213,20317844, \\
23702670,20496777,16211549, \\
17749106,22708943,88502, \\
43853,3010341\end{array}$ & 39.75832 & 45.236145 & -6.7 \\
\hline ZINC32922255 & 42960162,40159786 & 40.79816 & 43.46231 & -6.7 \\
\hline ZINC19790507 & $\begin{array}{l}3303636,675305,6920060, \\
3296004,3129060\end{array}$ & 39.869343 & 44.306328 & -6.7 \\
\hline ZINC41378161 & 53532662,52142760 & 40.995354 & 43.877476 & -6.7 \\
\hline ZINC04901110 & 1416244,6860800 & 41.068855 & 44.459133 & -6.7 \\
\hline ZINC02567292 & 12373187 & 39.792675 & 44.05333 & -6.7 \\
\hline ZINC71843930 & 56808613 & 41.026474 & 44.445858 & -6.6 \\
\hline ZINC49452908 & 53212200 & 40.173992 & 45.577507 & -6.6 \\
\hline ZINC04390787 & 2742693 & 40.95483 & 44.699516 & -6.6 \\
\hline ZINC16777002 & 7527589 & 40.438393 & 43.995045 & -6.6 \\
\hline ZINC11567959 & 24252607,42034680 & 40.670403 & 45.194748 & -6.6 \\
\hline
\end{tabular}




\begin{tabular}{|c|c|c|c|c|}
\hline ZINC05180285 & 941709,40511061 & 40.300545 & 43.435253 & -6.6 \\
\hline ZINC58217552 & 51091071 & 40.22932 & 44.303207 & -6.6 \\
\hline ZINC06557199 & 54708982 & 40.150566 & 43.90102 & -6.6 \\
\hline ZINC58635514 & 52562953 & 39.710205 & 43.92388 & -6.6 \\
\hline ZINC06493837 & 15998828 & 40.274014 & 44.346367 & -6.6 \\
\hline ZINC08653548 & 369641 & 40.020134 & 42.773804 & -6.6 \\
\hline ZINC13056335 & 25691431 & 41.13891 & 44.59531 & -6.6 \\
\hline ZINC13056330 & 25691426 & 41.139038 & 45.549316 & -6.6 \\
\hline ZINC72470337 & 56909147 & 39.267113 & 42.370586 & -6.6 \\
\hline ZINC05010607 & 7454182,16461774 & 40.011253 & 43.376602 & -6.6 \\
\hline ZINC19826774 & $\begin{array}{l}44162731,6859121,931772,695537 \\
5\end{array}$ & 41.055458 & 43.835983 & -6.6 \\
\hline ZINC04744605 & 2885921,7343806 & 41.421127 & 44.346497 & -6.6 \\
\hline ZINC40177522 & 46398601 & 40.384502 & 44.37789 & -6.6 \\
\hline ZINC65417585 & 50967677 & 39.57104 & 45.68682 & -6.6 \\
\hline ZINC63847854 & 46988048 & 40.11708 & 44.404423 & -6.6 \\
\hline ZINC44979262 & 45834844 & 41.44642 & 44.78051 & -6.6 \\
\hline ZINC12514327 & 2154007 & 40.300606 & 43.045155 & -6.6 \\
\hline ZINC57332633 & \begin{tabular}{|l|l}
52430445,50896025 \\
\end{tabular} & 40.93511 & 43.89788 & -6.6 \\
\hline ZINC66738332 & 53612698 & 41.233845 & 44.38163 & -6.6 \\
\hline ZINC16768225 & 51567386 & 41.149525 & 44.804165 & -6.6 \\
\hline ZINC57731629 & 46950637 & 41.390785 & 44.431652 & -6.6 \\
\hline ZINC64979532 & 53094879 & 40.628998 & 44.565502 & -6.6 \\
\hline ZINC04947632 & 54684645 & 40.1115 & 43.121742 & -6.6 \\
\hline ZINC01692038 & 267209 & 40.26581 & 44.95164 & -6.6 \\
\hline ZINC11848667 & \begin{tabular}{|l|l|}
42220317,16641557 \\
\end{tabular} & 40.93594 & 44.520943 & -6.6 \\
\hline ZINC02595875 & $\begin{array}{l}46924660,5630945,3793330, \\
4922013,5422342\end{array}$ & 39.994225 & 42.742615 & -6.6 \\
\hline ZINC16430939 & 51561981 & 39.916195 & 44.60559 & -6.6 \\
\hline ZINC01921678 & $1649279,1649280,54677700$ & 40.361755 & 45.33475 & -6.6 \\
\hline ZINC71915779 & 56806517 & 40.999252 & 44.859314 & -6.6 \\
\hline ZINC00290737 & $790395,2861871,40659356$ & 39.91627 & 44.54332 & -6.6 \\
\hline ZINC35291317 & 51685318,43811812 & 41.168884 & 44.98601 & -6.6 \\
\hline ZINC00039127 & 626452 & 40.99941 & 43.543266 & -6.6 \\
\hline ZINC71284481 & 56761350 & 39.675266 & 43.739925 & -6.6 \\
\hline ZINC19170617 & 706369,28083367 & 41.11527 & 43.78942 & -6.6 \\
\hline ZINC29112748 & 36287381 & 41.159164 & 44.08145 & -6.6 \\
\hline ZINC04920169 & $5390933,6098063,6738301$ & 40.85926 & 43.039734 & -6.6 \\
\hline ZINC00290736 & $790394,2861871,40659355$ & 39.942863 & 44.54332 & -6.6 \\
\hline ZINC28771051 & 44278292,12677785 & 39.591328 & 45.85649 & -6.6 \\
\hline ZINC58268733 & 51107858 & 41.28896 & 44.2921 & -6.6 \\
\hline ZINC38525946 & $70545108,70053411,10332676$ & 40.427338 & 44.71766 & -6.6 \\
\hline ZINC71920427 & 56806760 & 39.710438 & 43.235413 & -6.6 \\
\hline ZINC05759664 & $6330631,354148,54611155$ & 40.1734 & 44.58076 & -6.6 \\
\hline ZINC25804243 & 33078353,45801776 & 41.446304 & 44.44932 & -6.6 \\
\hline ZINC72164999 & 56900392 & 39.835808 & 45.15194 & -6.6 \\
\hline ZINC21301765 & 43825935 & 40.243305 & 44.54619 & -6.6 \\
\hline ZINC03855445 & 40467723,1249738 & 40.29774 & 43.435158 & -6.6 \\
\hline ZINC05937334 & 7831090,4780172 & 41.14629 & 43.0697 & -6.6 \\
\hline ZINC69772497 & 53603587 & 41.08943 & 44.795105 & -6.6 \\
\hline ZINC69594469 & 53585130 & 41.11301 & 45.06456 & -6.6 \\
\hline ZINC40285212 & \begin{tabular}{|l|l|}
51972955,49655629 \\
\end{tabular} & 40.262997 & 43.364994 & -6.6 \\
\hline ZINC07814381 & \begin{tabular}{|l|l}
8934005,16278585 \\
\end{tabular} & 40.740433 & 43.876213 & -6.6 \\
\hline ZINC20027495 & 43822923 & 41.164825 & 44.08831 & -6.6 \\
\hline ZINC69705422 & 53597585 & 41.16958 & 44.6481 & -6.6 \\
\hline ZINC65119350 & 53134915 & 40.66046 & 43.043545 & -6.6 \\
\hline ZINC04389524 & \begin{tabular}{|l|}
2739392,11890954 \\
\end{tabular} & 40.208652 & 43.820095 & -6.6 \\
\hline
\end{tabular}




\begin{tabular}{|c|c|c|c|c|}
\hline ZINC55244849 & 52390230,46991359 & 40.259758 & 43.38574 & -6.6 \\
\hline ZINC36358635 & 51719299,45493229 & 39.911175 & 44.338913 & -6.6 \\
\hline ZINC05074919 & $1256184,2978361,51409669$ & 41.09183 & 43.454197 & -6.6 \\
\hline ZINC21215728 & 29370671 & 39.755836 & 44.56862 & -6.6 \\
\hline ZINC04025636 & 3850470,7075936 & 41.15603 & 45.557354 & -6.6 \\
\hline ZINC28881381 & 44438506 & 39.53872 & 42.85827 & -6.6 \\
\hline ZINC16158817 & 2780359,26985520 & 39.86737 & 45.893852 & -6.6 \\
\hline ZINC04291283 & 7172352 & 40.282177 & 42.98033 & -6.6 \\
\hline ZINC02493965 & 53212143 & 40.37741 & 43.816635 & -6.6 \\
\hline ZINC35425564 & 51691854,45783601 & 40.84061 & 44.297276 & -6.6 \\
\hline ZINC12997732 & 25658380,42932171 & 39.99275 & 42.757183 & -6.6 \\
\hline ZINC71802807 & 56811890 & 41.193012 & 44.763756 & -6.6 \\
\hline ZINC32501651 & 39734340 & 40.20844 & 44.357758 & -6.6 \\
\hline ZINC72474808 & 56911228 & 41.378933 & 45.6184 & -6.6 \\
\hline ZINC46494409 & 56796159 & 41.44771 & 44.488743 & -6.6 \\
\hline ZINC02787381 & 4774548 & 41.14985 & 45.22752 & -6.6 \\
\hline ZINC03272897 & 2388949,7041392 & 40.20951 & 43.37315 & -6.6 \\
\hline ZINC45796197 & 50742686 & 41.14541 & 45.076916 & -6.6 \\
\hline ZINC14200555 & 17470959,25988568 & 41.176895 & 43.941383 & -6.6 \\
\hline ZINC14200556 & 17470959,25988569 & 41.178017 & 44.047897 & -6.6 \\
\hline ZINC05733697 & 3004499,3481981 & 39.691414 & 43.02753 & -6.6 \\
\hline ZINC18143324 & 379751 & 39.95965 & 43.74528 & -6.6 \\
\hline ZINC78527036 & 60266336 & 40.814667 & 44.4432 & -6.6 \\
\hline ZINC71284482 & 56761350 & 39.98765 & 43.350643 & -6.6 \\
\hline ZINC41329118 & 12079466 & 40.96562 & 44.272934 & -6.6 \\
\hline ZINC04477324 & 16460308 & 41.180294 & 45.97981 & -6.5 \\
\hline ZINC48373930 & 53499486 & 41.244602 & 43.074013 & -6.5 \\
\hline ZINC16958045 & 372012 & 40.02178 & 42.767296 & -6.5 \\
\hline ZINC17327366 & 51228978 & 41.161137 & 44.421375 & -6.5 \\
\hline ZINC23251761 & 30957695 & 41.26078 & 44.04589 & -6.5 \\
\hline ZINC12592602 & 25381120,16379958 & 41.16018 & 44.28267 & -6.5 \\
\hline ZINC54097406 & 800340,33193285 & 39.5969 & 42.90042 & -6.5 \\
\hline ZINC13638811 & 6881904,6746387 & 39.65216 & 42.055943 & -6.5 \\
\hline ZINC69561167 & 53579638 & 41.11315 & 44.18214 & -6.5 \\
\hline ZINC36533612 & 51777265,45933594 & 39.963036 & 46.000774 & -6.5 \\
\hline ZINC05687033 & 40555573,784829 & 40.97496 & 44.600292 & -6.5 \\
\hline ZINC33332573 & 1402697,6404132 & 39.962166 & 43.956146 & -6.5 \\
\hline ZINC69492432 & 53571729 & 40.811737 & 45.305443 & -6.5 \\
\hline ZINC32205051 & 39439603 & 40.96821 & 45.036625 & -6.5 \\
\hline ZINC13467928 & 2728468,25772211 & 41.022163 & 43.339127 & -6.5 \\
\hline ZINC13268310 & 46956644,51519946 & 40.799877 & 42.947266 & -6.5 \\
\hline ZINC66469577 & 53609189 & 40.75694 & 45.309772 & -6.5 \\
\hline ZINC56683471 & 1400053 & 39.65805 & 43.958397 & -6.5 \\
\hline ZINC06752347 & 6625427 & 41.17549 & 43.364006 & -6.5 \\
\hline ZINC24772118 & 42948121,32085115 & 40.024178 & 43.89997 & -6.5 \\
\hline ZINC45928736 & 54729768 & 40.096287 & 43.741634 & -6.5 \\
\hline ZINC45795895 & 50742566 & 40.738045 & 44.645252 & -6.5 \\
\hline ZINC01384403 & $51380112,3743908,1472945$ & 41.206238 & 44.833897 & -6.5 \\
\hline ZINC82752549 & 66425100 & 39.862 & 44.439945 & -6.5 \\
\hline ZINC06922344 & 3645565 & 39.11199 & 44.964844 & -6.5 \\
\hline ZINC67954603 & 56758397 & 40.163742 & 43.25297 & -6.5 \\
\hline ZINC49453721 & 53212826 & 41.165165 & 44.25013 & -6.5 \\
\hline ZINC05720648 & $3498272,16413292,28906979$ & 41.20493 & 45.215427 & -6.5 \\
\hline ZINC69869889 & 53612449 & 40.80695 & 44.856823 & -6.5 \\
\hline ZINC72237458 & 66487851 & 39.343174 & 45.20804 & -6.5 \\
\hline ZINC44913250 & 52210033,45813011 & 40.866898 & 44.592884 & -6.5 \\
\hline ZINC05625917 & 6891783,1402697 & 39.973297 & 43.95407 & -6.5 \\
\hline
\end{tabular}




\begin{tabular}{|c|c|c|c|c|}
\hline ZINC58268734 & 51107858 & 41.283077 & 44.30179 & -6.5 \\
\hline ZINC67459936 & 56701808 & 40.917534 & 44.204636 & -6.5 \\
\hline ZINC67730164 & 3743379 & 39.889256 & 43.67275 & -6.5 \\
\hline ZINC40556025 & 45834938 & 39.972176 & 43.0716 & -6.5 \\
\hline ZINC22159500 & 30064388,16780466 & 39.57138 & 44.74215 & -6.5 \\
\hline ZINC06265149 & 4381944 & 39.114407 & 44.976585 & -6.5 \\
\hline ZINC54203587 & 47059800 & 41.44624 & 44.40934 & -6.5 \\
\hline ZINC72168306 & 56914017 & 40.073765 & 43.30165 & -6.5 \\
\hline ZINC23107049 & 30835304,42952393 & 41.390057 & 44.469704 & -6.5 \\
\hline ZINC49453933 & 53212990 & 40.198204 & 45.70333 & -6.5 \\
\hline ZINC02516924 & 16218650,114997 & 40.97763 & 42.810528 & -6.5 \\
\hline ZINC71284484 & 56761350 & 39.67537 & 43.817894 & -6.5 \\
\hline ZINC35475871 & 52998509 & 40.25786 & 45.71839 & -6.5 \\
\hline ZINC02150471 & 54682647,1801066 & 41.327595 & 43.90723 & -6.5 \\
\hline ZINC11981876 & 24265455,42331344 & 38.87389 & 44.1176 & -6.5 \\
\hline ZINC31775837 & 38999863 & 40.899956 & 44.820927 & -6.5 \\
\hline ZINC03623428 & $7044599,194594,194595$ & 40.79871 & 43.669525 & -6.5 \\
\hline ZINC08764996 & 40816422,16409105 & 40.047466 & 45.118553 & -6.5 \\
\hline ZINC32205034 & 39439583 & 41.124702 & 45.007782 & -6.5 \\
\hline ZINC05672486 & 6826657 & 40.96119 & 43.600685 & -6.5 \\
\hline ZINC05625903 & 1400053,6891139 & 39.658573 & 43.956146 & -6.5 \\
\hline ZINC52099094 & 62374385 & 40.185585 & 44.782246 & -6.5 \\
\hline ZINC69057864 & 53609983 & 41.150127 & 43.171112 & -6.5 \\
\hline ZINC07865651 & 8985446 & 39.798283 & 44.027798 & -6.5 \\
\hline ZINC21779183 & 46257767 & 40.255554 & 43.89515 & -6.5 \\
\hline ZINC44890584 & 45799935 & 41.17872 & 43.598385 & -6.5 \\
\hline ZINC39320601 & 45156807 & 40.44878 & 45.466347 & -6.5 \\
\hline ZINC20375744 & 28896350 & 39.79305 & 44.441612 & -6.5 \\
\hline ZINC53994859 & 50877734 & 39.66625 & 45.20865 & -6.5 \\
\hline ZINC57908856 & 45834942 & 40.865215 & 42.69451 & -6.5 \\
\hline ZINC39276912 & 12736944 & 40.996853 & 43.515163 & -6.5 \\
\hline ZINC62719770 & 54735265 & 39.706814 & 43.358288 & -6.5 \\
\hline ZINC32113361 & 39363723 & 40.853245 & 43.597416 & -6.5 \\
\hline ZINC64871591 & 53263258 & 40.632866 & 43.328907 & -6.5 \\
\hline ZINC08969429 & 5741848 & 40.962204 & 42.849022 & -6.5 \\
\hline ZINC34927270 & 25220687 & 40.822216 & 44.880955 & -6.5 \\
\hline ZINC36354218 & 51717511,45491440 & 41.17018 & 45.06528 & -6.5 \\
\hline ZINC71751939 & 56873088 & 39.509354 & 45.417313 & -6.5 \\
\hline ZINC03306864 & 2422596,2997552 & 41.150127 & 44.326748 & -6.5 \\
\hline ZINC65470515 & 50977017 & 40.173965 & 43.379505 & -6.5 \\
\hline ZINC31156947 & 45359149,38348510 & 39.651566 & 43.931957 & -6.5 \\
\hline ZINC36895314 & 51885107,43142054 & 40.26188 & 44.95607 & -6.5 \\
\hline ZINC63847112 & 46987300 & 40.91053 & 44.772736 & -6.5 \\
\hline ZINC05955961 & $17598581,5417893,6746172$ & 40.847946 & 43.767124 & -6.5 \\
\hline ZINC20737134 & 29136370,42652492 & 41.313103 & 44.01524 & -6.5 \\
\hline ZINC00977538 & $1207560,53450600,1776679$ & 39.925236 & 43.97891 & -6.4 \\
\hline ZINC03098185 & $44784287,3792316,51056593$ & 41.08522 & 45.255222 & -6.4 \\
\hline ZINC03098183 & $44726009,7034791,5132208$ & 41.085182 & 45.27127 & -6.4 \\
\hline ZINC13533896 & $51522968,25794079,18524101$ & 40.96038 & 44.44497 & -6.4 \\
\hline ZINC14140764 & 17453252,25959990 & 41.122356 & 44.95247 & -6.4 \\
\hline ZINC65433755 & 53504152 & 41.260643 & 43.674706 & -6.4 \\
\hline ZINC71285470 & 60181486 & 40.509995 & 43.706066 & -6.4 \\
\hline ZINC13687889 & 1627636,25845894 & 40.997723 & 43.381645 & -6.4 \\
\hline ZINC36293971 & 51715437,44669185 & 40.07161 & 43.92469 & -6.4 \\
\hline ZINC03205234 & 2327408 & 41.03608 & 44.217606 & -6.4 \\
\hline ZINC47843889 & 52271245,47002720 & 40.363163 & 43.478584 & -6.4 \\
\hline ZINC35655300 & 51698501,43840602 & 41.09387 & 44.55233 & -6.4 \\
\hline
\end{tabular}




\begin{tabular}{|c|c|c|c|c|}
\hline ZINC03073672 & 44784286,3732502 & 41.201305 & 45.38539 & -6.4 \\
\hline ZINC04451125 & 3156299 & 41.125137 & 44.745167 & -6.4 \\
\hline ZINC39344071 & 12941201 & 41.115425 & 45.04417 & -6.4 \\
\hline ZINC49714545 & 61557562 & 39.94397 & 43.678596 & -6.4 \\
\hline ZINC06357324 & 69081847,3328703 & 40.16957 & 45.06169 & -6.4 \\
\hline ZINC71883397 & 56782472 & 41.20436 & 44.942314 & -6.4 \\
\hline ZINC18149165 & 6953102,892583 & 41.054737 & 45.118683 & -6.4 \\
\hline ZINC82752548 & 66425100 & 39.860565 & 44.43985 & -6.4 \\
\hline ZINC14953365 & $\begin{array}{l}\text { 9948293,9828904,69936192, } \\
18770508,5722,67243513, \\
26317648,69823923\end{array}$ & 41.22032 & 44.498768 & -6.4 \\
\hline ZINC22699378 & 30545234 & 40.98621 & 43.472424 & -6.4 \\
\hline ZINC04185042 & 3146815,5767795 & 40.2157 & 44.25058 & -6.4 \\
\hline ZINC03203056 & 2326484,11865101 & 40.04765 & 43.219437 & -6.4 \\
\hline ZINC33633577 & 51664706,3521834 & 40.820984 & 43.34305 & -6.4 \\
\hline ZINC40448209 & 51980210,51064053 & 39.87304 & 44.05443 & -6.4 \\
\hline ZINC11567960 & 24252607,42034682 & 40.67047 & 45.185276 & -6.4 \\
\hline ZINC72171581 & 56916599 & 39.514698 & 45.675934 & -6.4 \\
\hline ZINC00038345 & 6920095,675533 & 41.168873 & 44.474022 & -6.4 \\
\hline ZINC12925316 & 25609398 & 40.967155 & 43.676502 & -6.4 \\
\hline ZINC67172770 & 53337822 & 40.861557 & 45.188828 & -6.4 \\
\hline ZINC49456054 & 53214187 & 40.18933 & 45.288742 & -6.4 \\
\hline ZINC69150522 & 53500081 & 41.08583 & 45.117767 & -6.4 \\
\hline ZINC24772111 & 42948121,32085108 & 40.02493 & 43.97027 & -6.4 \\
\hline ZINC72126600 & 56883916 & 40.881012 & 45.748264 & -6.4 \\
\hline ZINC71415810 & 56861968 & 40.860836 & 44.746243 & -6.4 \\
\hline ZINC67756448 & 56708231 & 41.137947 & 43.43016 & -6.4 \\
\hline ZINC06750930 & 6625279 & 41.18582 & 45.849716 & -6.4 \\
\hline ZINC14135843 & 25957888,16582477 & 39.512917 & 44.5063 & -6.4 \\
\hline ZINC14135841 & 25957887,16582477 & 39.501553 & 43.083534 & -6.4 \\
\hline ZINC16769935 & 4736988 & 41.03062 & 44.74259 & -6.4 \\
\hline ZINC83501969 & 65143072 & 41.147877 & 43.173027 & -6.4 \\
\hline ZINC04977384 & $\begin{array}{l}44144470,45157528,88589, \\
60160951,161556,90291, \\
3449165,75869,75868, \\
56842808\end{array}$ & 39.995705 & 45.39764 & -6.4 \\
\hline ZINC77286241 & 69409602 & 39.880962 & 44.64309 & -6.4 \\
\hline ZINC57291646 & 40989757 & 39.658943 & 44.77335 & -6.4 \\
\hline ZINC34154090 & 13781523 & 39.31286 & 45.352013 & -6.4 \\
\hline ZINC04404251 & $\begin{array}{l}46924602,44664425,4368318, \\
40486055,908101\end{array}$ & 39.99477 & 43.69833 & -6.4 \\
\hline ZINC01393470 & 6986890,1480510 & 41.18998 & 45.712914 & -6.4 \\
\hline ZINC67730170 & 40523422,3743379 & 39.889027 & 45.696404 & -6.4 \\
\hline ZINC01622792 & 365360 & 40.887325 & 45.466743 & -6.4 \\
\hline ZINC02388774 & 1990881 & 39.114998 & 43.93885 & -6.4 \\
\hline ZINC18107324 & 27839312,698147 & 41.173187 & 43.49544 & -6.4 \\
\hline ZINC34929140 & 42647325 & 41.102676 & 42.436424 & -6.4 \\
\hline ZINC31869825 & 39086256,52983716 & 40.79571 & 44.34741 & -6.4 \\
\hline ZINC31869824 & 39086254,52983716 & 40.794983 & 45.03525 & -6.4 \\
\hline ZINC06562282 & 40626144 & 40.11409 & 43.376892 & -6.4 \\
\hline ZINC20282609 & 28819304,42953282 & 41.060852 & 45.36058 & -6.4 \\
\hline ZINC72122290 & 56882398 & 40.072514 & 43.767284 & -6.4 \\
\hline ZINC16904052 & 27408513 & 39.83057 & 43.829185 & -6.4 \\
\hline ZINC03306862 & 2422593,2997552 & 41.150127 & 44.589485 & -6.4 \\
\hline ZINC01651205 & 390576 & 41.158966 & 44.34664 & -6.4 \\
\hline ZINC07011373 & $40660633,4728735,40660641$ & 40.169502 & 44.541786 & -6.4 \\
\hline ZINC22211942 & 30112821,16788172 & 40.41411 & 45.132156 & -6.4 \\
\hline
\end{tabular}




\begin{tabular}{|c|c|c|c|c|}
\hline ZINC39394609 & $25368022,25368023,45193959$ & 40.81727 & 44.16723 & -6.4 \\
\hline ZINC13519811 & $971628,4695070,51522865$ & 40.96246 & 43.59552 & -6.4 \\
\hline ZINC63847114 & 46987300 & 41.04287 & 44.6174 & -6.4 \\
\hline ZINC23731230 & 9350392,9350393 & 40.07229 & 43.418655 & -6.4 \\
\hline ZINC03652507 & 5767942,3147474 & 40.216927 & 44.246204 & -6.4 \\
\hline ZINC13682850 & $\begin{array}{l}25844796,6796204,9626620, \\
5688263\end{array}$ & 39.900604 & 42.895603 & -6.4 \\
\hline ZINC18240650 & $6239122,44662156,51580561$ & 40.999584 & 43.011024 & -6.4 \\
\hline ZINC72472963 & 56909498 & 39.683434 & 44.199806 & -6.4 \\
\hline ZINC08494803 & 51235388 & 40.214767 & 45.305687 & -6.4 \\
\hline ZINC39389368 & 51902006,44615706 & 40.77066 & 45.86177 & -6.4 \\
\hline ZINC08745254 & 17420810,40808844 & 41.043545 & 43.739223 & -6.4 \\
\hline ZINC08745256 & 17420810,40808848 & 41.04369 & 43.71038 & -6.4 \\
\hline ZINC00894371 & 1149027,44161780 & 41.16253 & 44.34079 & -6.4 \\
\hline ZINC06505131 & $45045543,4400437,54696572$ & 40.116314 & 43.06507 & -6.4 \\
\hline ZINC67974657 & 56722445 & 39.683434 & 45.5726 & -6.4 \\
\hline ZINC65748828 & 11507097 & 41.285866 & 44.641968 & -6.3 \\
\hline ZINC06553506 & 598216,6952553 & 41.389786 & 44.754837 & -6.3 \\
\hline ZINC03851874 & 7057477 & 41.14303 & 43.898373 & -6.3 \\
\hline ZINC39389354 & 44615699 & 40.306442 & 45.305992 & -6.3 \\
\hline ZINC10813320 & 17449579,41676021 & 41.440243 & 43.719513 & -6.3 \\
\hline ZINC65512793 & 53541886 & 40.151993 & 43.775677 & -6.3 \\
\hline ZINC49453096 & 53212343 & 39.330704 & 45.64057 & -6.3 \\
\hline ZINC04350612 & 2187384 & 39.765938 & 44.28156 & -6.3 \\
\hline ZINC00207539 & $754190,1636766,655766,3275762$ & 41.218197 & 44.43971 & -6.3 \\
\hline ZINC19894290 & 44120591 & 39.79563 & 43.22607 & -6.3 \\
\hline ZINC62208626 & 57504584 & 41.435566 & 45.633133 & -6.3 \\
\hline ZINC67804670 & 56715879 & 40.19712 & 45.00996 & -6.3 \\
\hline ZINC10813314 & 17449579,41676016 & 41.446968 & 44.49954 & -6.3 \\
\hline ZINC72472962 & 56909498 & 39.684807 & 45.071865 & -6.3 \\
\hline ZINC24924301 & 24249823 & 40.300175 & 44.60073 & -6.3 \\
\hline ZINC01304621 & 6985255,1419051 & 40.0984 & 46.110924 & -6.3 \\
\hline ZINC22121593 & 30029700 & 41.04555 & 44.1081 & -6.3 \\
\hline ZINC11865964 & 24280945,42234754 & 41.170223 & 43.665173 & -6.3 \\
\hline ZINC67172750 & 53337403 & 39.945957 & 45.212948 & -6.3 \\
\hline ZINC41154199 & 52112370 & 41.34529 & 43.898354 & -6.3 \\
\hline ZINC83237580 & 53270535 & 39.66676 & 43.80892 & -6.3 \\
\hline ZINC49453722 & 53212827 & 41.167374 & 44.245087 & -6.3 \\
\hline ZINC00394847 & $\begin{array}{l}97423,6950771,67791348,5470665 \\
0\end{array}$ & 40.044876 & 45.16338 & -6.3 \\
\hline ZINC22134289 & 18131193 & 41.446815 & 43.95544 & -6.3 \\
\hline ZINC34752479 & 59270622,15866125 & 41.081944 & 43.448566 & -6.3 \\
\hline ZINC26421177 & 33676879 & 41.13692 & 44.80306 & -6.3 \\
\hline ZINC19528164 & $\begin{array}{l}\text { 11948889,51595456,7436843, } \\
1439217\end{array}$ & 39.619442 & 44.727608 & -6.3 \\
\hline ZINC65734521 & 53434980 & 40.339848 & 45.467022 & -6.3 \\
\hline ZINC06322563 & 4196516,51421708 & 41.09118 & 45.223354 & -6.3 \\
\hline ZINC08306619 & 9429890 & 40.015526 & 45.098183 & -6.3 \\
\hline ZINC39951953 & 45072150 & 41.042713 & 45.28505 & -6.3 \\
\hline ZINC07155632 & 8272268 & 41.01697 & 44.480873 & -6.3 \\
\hline ZINC05926108 & $\begin{array}{l}46924602,44664425,40572196, \\
4368318,908102\end{array}$ & 39.995216 & 43.70278 & -6.3 \\
\hline ZINC14180450 & 17460267,25980312 & 40.02864 & 42.546307 & -6.3 \\
\hline ZINC05539376 & 5343032,3750277 & 39.75869 & 45.15404 & -6.3 \\
\hline ZINC39127823 & 14700274 & 39.59632 & 44.732117 & -6.3 \\
\hline ZINC36893222 & 51885016,43140166 & 41.217987 & 45.77922 & -6.3 \\
\hline ZINC21984102 & 29920871 & 39.491222 & 44.946335 & -6.3 \\
\hline
\end{tabular}




\begin{tabular}{|c|c|c|c|c|}
\hline ZINC71840950 & 60395994 & 40.817963 & 45.816814 & -6.3 \\
\hline ZINC26421185 & 33676889 & 41.085403 & 44.802906 & -6.3 \\
\hline ZINC49453739 & 53212844 & 41.16589 & 43.92414 & -6.3 \\
\hline ZINC36290969 & 67425346,58551898 & 41.07708 & 43.61805 & -6.3 \\
\hline ZINC00381817 & 847773,6949735 & 40.052826 & 44.066128 & -6.3 \\
\hline ZINC32112368 & 39362101 & 41.022354 & 44.402893 & -6.3 \\
\hline ZINC22097835 & 30016350,30016351 & 41.21622 & 44.54199 & -6.3 \\
\hline ZINC69808533 & 53606593 & 41.24533 & 43.24744 & -6.3 \\
\hline ZINC03399141 & $40460221,2976315,866391$ & 39.94745 & 44.237274 & -6.3 \\
\hline ZINC49449720 & 49736831 & 40.86983 & 44.62503 & -6.3 \\
\hline ZINC07948397 & 16187931,9784498 & 41.034515 & 44.167618 & -6.3 \\
\hline ZINC21030664 & 20958637 & 40.95994 & 44.51411 & -6.3 \\
\hline ZINC02526610 & 4773801 & 41.005585 & 44.3583 & -6.3 \\
\hline ZINC20134313 & 17609308 & 41.27944 & 44.699913 & -6.3 \\
\hline ZINC33332432 & $51415180,6404063,1400053$ & 39.962616 & 43.95614 & -6.3 \\
\hline ZINC67792716 & 50998460 & 40.031643 & 45.157333 & -6.3 \\
\hline ZINC72433520 & 56882756 & 39.858612 & 45.0929 & -6.3 \\
\hline ZINC20282605 & 28819302,42953282 & 41.061203 & 45.33052 & -6.3 \\
\hline ZINC08050106 & 53536377 & 40.944946 & 44.91083 & -6.3 \\
\hline ZINC67974655 & 56722445 & 39.684425 & 45.532536 & -6.3 \\
\hline ZINC08745255 & 17420810,40808846 & 41.047443 & 43.811954 & -6.3 \\
\hline ZINC49454590 & 53213545 & 40.786484 & 43.873215 & -6.3 \\
\hline ZINC10166701 & 18575873 & 39.928295 & 44.67329 & -6.3 \\
\hline ZINC10166700 & 18575873 & 39.928295 & 44.737617 & -6.3 \\
\hline ZINC36973975 & 43170874 & 39.943607 & 45.485542 & -6.3 \\
\hline ZINC31156942 & 45359149,38348503 & 39.524284 & 45.365944 & -6.3 \\
\hline ZINC05611551 & 40549187,3655274 & 40.305862 & 43.949036 & -6.3 \\
\hline ZINC13268309 & 46956644,51519945 & 40.799488 & 43.198174 & -6.3 \\
\hline ZINC13686987 & 17595129,25845755 & 40.96184 & 43.607986 & -6.3 \\
\hline ZINC00117967 & 2809568,6926734 & 40.96543 & 42.747887 & -6.3 \\
\hline ZINC49465461 & 47469138 & 41.199112 & 44.658176 & -6.2 \\
\hline ZINC04010271 & 51398486 & 39.27809 & 43.68614 & -6.2 \\
\hline ZINC40496948 & 45808301 & 40.169956 & 43.533463 & -6.2 \\
\hline ZINC49455889 & 53214116 & 40.169006 & 44.84839 & -6.2 \\
\hline ZINC66351901 & 46863990 & 40.962097 & 43.44311 & -6.2 \\
\hline ZINC22159497 & $30064384,16780466,57367593$ & 39.57286 & 44.74511 & -6.2 \\
\hline ZINC26479688 & 33752698 & 40.75298 & 43.16655 & -6.2 \\
\hline ZINC35288556 & 51684378,12627621 & 39.852325 & 43.89627 & -6.2 \\
\hline ZINC61966104 & 52903937 & 40.89099 & 45.92365 & -6.2 \\
\hline ZINC33962936 & 9795476 & 40.150246 & 43.592297 & -6.2 \\
\hline ZINC53994335 & 49757326 & 40.8136 & 44.05165 & -6.2 \\
\hline ZINC80345726 & 60173992 & 41.148293 & 43.728466 & -6.2 \\
\hline ZINC00154359 & 2736213,6931036 & 40.879337 & 45.709965 & -6.2 \\
\hline ZINC06928573 & 3352935 & 41.162 & 44.88033 & -6.2 \\
\hline ZINC67678969 & 50967371 & 39.539062 & 45.71933 & -6.2 \\
\hline ZINC14177876 & 25978871 & 40.00919 & 45.08052 & -6.2 \\
\hline ZINC29464187 & 36657888 & 40.980095 & 43.41285 & -6.2 \\
\hline ZINC39394607 & $25368017,25368018,45193959$ & 40.81803 & 44.06431 & -6.2 \\
\hline ZINC45922306 & 46507189 & 41.44127 & 44.541092 & -6.2 \\
\hline ZINC48300143 & 47023794 & 40.980553 & 42.68425 & -6.2 \\
\hline ZINC33616566 & 3498272 & 41.203266 & 43.37329 & -6.2 \\
\hline ZINC33616565 & 3498272 & 41.202995 & 43.37243 & -6.2 \\
\hline ZINC02516125 & 7015690 & 40.052475 & 44.351723 & -6.2 \\
\hline ZINC04107268 & 5083854 & 41.093292 & 44.715687 & -6.2 \\
\hline ZINC37967220 & 61001220 & 41.034588 & 45.038044 & -6.2 \\
\hline ZINC02216243 & $3334457,1849004,51383378$ & 40.626022 & 43.778202 & -6.2 \\
\hline ZINC12367733 & 2825623,23252695 & 40.93774 & 44.919994 & -6.2 \\
\hline
\end{tabular}




\begin{tabular}{|c|c|c|c|c|}
\hline ZINC38024004 & 54899033 & 40.19124 & 44.777306 & -6.2 \\
\hline ZINC19878125 & $28422986,6834655,16470641$ & 40.1112 & 43.79232 & -6.2 \\
\hline ZINC72233006 & 66524886 & 40.29144 & 45.13842 & -6.2 \\
\hline ZINC04218759 & 7131365,8829768 & 39.84597 & 45.96831 & -6.2 \\
\hline ZINC04025637 & 377796 & 39.43392 & 43.77806 & -6.2 \\
\hline ZINC22828966 & 51621944,45923966 & 39.60003 & 43.54102 & -6.2 \\
\hline ZINC13366099 & 20855176 & 40.782818 & 44.962135 & -6.2 \\
\hline ZINC42784397 & 52181769,49762949 & 39.567932 & 44.711124 & -6.2 \\
\hline ZINC44979279 & 45834850 & 41.446613 & 43.3396 & -6.2 \\
\hline ZINC13125365 & 5707305,2766325 & 40.296913 & 43.353867 & -6.2 \\
\hline ZINC13125366 & 2766278,5707297 & 40.789814 & 43.353905 & -6.2 \\
\hline ZINC15081711 & 26413946,16765383 & 40.876545 & 45.304432 & -6.2 \\
\hline ZINC62591956 & 52909567,51042760 & 40.773212 & 44.65783 & -6.2 \\
\hline ZINC00874374 & 1133439 & 40.0632 & 44.27064 & -6.2 \\
\hline ZINC17327370 & 51228978 & 41.16027 & 44.42125 & -6.2 \\
\hline ZINC67788131 & 56715498 & 41.024776 & 44.910713 & -6.2 \\
\hline ZINC47521245 & 52263622 & 39.602726 & 43.25753 & -6.2 \\
\hline ZINC05278177 & 5217553,7602495 & 41.193996 & 43.96765 & -6.2 \\
\hline ZINC36358916 & 51719501,45493449 & 41.248917 & 45.4601 & -6.2 \\
\hline ZINC71888013 & 56783689 & 40.813606 & 44.235943 & -6.2 \\
\hline ZINC67730168 & 3743379 & 39.889145 & 44.80775 & -6.2 \\
\hline ZINC13214989 & 5467938,378267 & 40.210594 & 45.23881 & -6.2 \\
\hline ZINC09954520 & 16392557 & 41.446148 & 44.629047 & -6.2 \\
\hline ZINC13215719 & 5468613,383914 & 41.144794 & 44.946404 & -6.2 \\
\hline ZINC01730409 & $77804,20371977,77805,21904465$ & 40.915436 & 44.41902 & -6.2 \\
\hline ZINC71802778 & 56811957 & 40.117367 & 44.46413 & -6.2 \\
\hline ZINC24055047 & 43049483,31536844 & 40.351357 & 44.230118 & -6.2 \\
\hline ZINC03331468 & 40459871,2115209 & 40.76651 & 44.48116 & -6.2 \\
\hline ZINC32628323 & 39870857,16781090 & 40.77827 & 45.61251 & -6.2 \\
\hline ZINC04323280 & 9583512,2805326 & 39.62407 & 45.26528 & -6.2 \\
\hline ZINC05458064 & 40537294,4126941 & 40.48515 & 43.390453 & -6.2 \\
\hline ZINC25138407 & 32449763 & 41.447453 & 44.61145 & -6.2 \\
\hline ZINC05196462 & 16187931,9075774 & 41.035667 & 44.5447 & -6.2 \\
\hline ZINC15987484 & 1478662,26891711 & 41.192078 & 43.317287 & -6.1 \\
\hline ZINC05809207 & $6763151,628447,6364420$ & 39.88006 & 43.04059 & -6.1 \\
\hline ZINC12592606 & 25381126,16379958 & 41.159313 & 44.817932 & -6.1 \\
\hline ZINC01742588 & 5006471 & 41.289867 & 44.840454 & -6.1 \\
\hline ZINC62152035 & 52908275 & 41.364346 & 45.959587 & -6.1 \\
\hline ZINC53993710 & 33677924 & 40.816174 & 44.465282 & -6.1 \\
\hline ZINC20272147 & 28811183 & 39.964085 & 43.338917 & -6.1 \\
\hline ZINC21953961 & 29893862,16770629 & 40.551888 & 45.79297 & -6.1 \\
\hline ZINC39414789 & 13175377 & 40.827858 & 44.208847 & -6.1 \\
\hline ZINC04370250 & 2825623,5716598 & 40.938805 & 44.821175 & -6.1 \\
\hline ZINC69596726 & 53585582 & 41.04707 & 43.172497 & -6.1 \\
\hline ZINC36293969 & 51715435,44669185 & 39.864113 & 43.782295 & -6.1 \\
\hline ZINC55393628 & 52399878,50982180 & 40.769157 & 44.475258 & -6.1 \\
\hline ZINC61957845 & 52903564 & 41.165436 & 43.650253 & -6.1 \\
\hline ZINC43827879 & 52194557,50853233 & 40.813248 & 43.522156 & -6.1 \\
\hline ZINC12954541 & 19256562,25630979 & 41.051273 & 45.996017 & -6.1 \\
\hline ZINC49409518 & 54733578 & 40.050976 & 43.25856 & -6.1 \\
\hline ZINC17327372 & 51228978 & 41.158936 & 44.42123 & -6.1 \\
\hline ZINC01384402 & 1472944,3743908 & 41.20348 & 44.80803 & -6.1 \\
\hline ZINC12367734 & 2825623,42555232 & 40.93741 & 44.908115 & -6.1 \\
\hline ZINC55271497 & 46992788 & 40.764732 & 44.498985 & -6.1 \\
\hline ZINC40313251 & $56738073,51975009,49652681$ & 39.485607 & 43.438885 & -6.1 \\
\hline ZINC66082366 & 51138340 & 40.81046 & 44.7059 & -6.1 \\
\hline ZINC67788568 & 56715550 & 40.430893 & 43.929787 & -6.1 \\
\hline
\end{tabular}




\begin{tabular}{|c|c|c|c|c|}
\hline ZINC00079370 & 700001,4137135 & 39.84794 & 44.650097 & -6.1 \\
\hline ZINC61957846 & 52903566 & 40.98916 & 43.655018 & -6.1 \\
\hline ZINC35305510 & 12493521 & 40.958736 & 44.679058 & -6.1 \\
\hline ZINC83237578 & 53270535 & 39.66676 & 45.53491 & -6.1 \\
\hline ZINC38027138 & 61010988 & 39.56251 & 44.099087 & -6.1 \\
\hline ZINC05415968 & 3316472 & 40.015514 & 44.342022 & -6.1 \\
\hline ZINC13111412 & 850989,25714113 & 41.139435 & 43.48034 & -6.1 \\
\hline ZINC18122183 & $27847605,4064138,671869$ & 41.487827 & 43.823185 & -6.1 \\
\hline ZINC44979276 & 45834850 & 41.447735 & 44.72222 & -6.1 \\
\hline ZINC16905445 & 27410063 & 40.29962 & 45.732803 & -6.1 \\
\hline ZINC49484609 & 49746607 & 41.193012 & 43.70901 & -6.1 \\
\hline ZINC12520656 & $25330898,16617625,51508987$ & 40.188995 & 43.42598 & -6.1 \\
\hline ZINC15973895 & 51553592,26885819 & 41.287636 & 45.735664 & -6.1 \\
\hline ZINC00144515 & 731146,6929823 & 40.98913 & 45.227325 & -6.1 \\
\hline ZINC79484753 & 60175510 & 41.23421 & 44.63672 & -6.1 \\
\hline ZINC12978605 & 42885474 & 39.946335 & 44.35338 & -6.1 \\
\hline ZINC32850508 & 40083967 & 40.073177 & 44.917843 & -6.1 \\
\hline ZINC07974999 & 9102255 & 41.11495 & 43.451927 & -6.1 \\
\hline ZINC71888012 & 56783689 & 40.813606 & 44.235943 & -6.1 \\
\hline ZINC35305505 & 42763690 & 40.958946 & 44.678802 & -6.1 \\
\hline ZINC38006771 & 61000883 & 41.179035 & 42.940426 & -6.1 \\
\hline ZINC62591944 & 52909561,51042752 & 39.77687 & 43.8422 & -6.1 \\
\hline ZINC83662598 & 64282205 & 41.16182 & 44.93859 & -6.1 \\
\hline ZINC24055049 & 43049483,31536845 & 40.352203 & 43.882233 & -6.1 \\
\hline ZINC08745253 & 17420810,40808842 & 41.03979 & 43.80775 & -6.1 \\
\hline ZINC02482737 & 40421544,1559055 & 41.18348 & 44.471275 & -6 \\
\hline ZINC00343084 & 549134 & 39.676693 & 45.262108 & -6 \\
\hline ZINC49452846 & 67454045,53212170 & 39.62512 & 43.907658 & -6 \\
\hline ZINC20427142 & 28935047 & 40.791916 & 44.666122 & -6 \\
\hline ZINC22065935 & $29986488,13450921,23722420$ & 40.05577 & 44.856457 & -6 \\
\hline ZINC38027874 & 61010427 & 41.10002 & 45.305634 & -6 \\
\hline ZINC23624019 & $31263527,16370863,41260388$ & 40.10224 & 43.065372 & -6 \\
\hline ZINC49449932 & 49736927 & 40.87009 & 44.624958 & -6 \\
\hline ZINC19200266 & $\begin{array}{l}\text { 6501902,7426773,28093677, } \\
54676379\end{array}$ & 39.81508 & 43.272026 & -6 \\
\hline ZINC06173363 & 2386023,40590929 & 40.920574 & 43.261135 & -6 \\
\hline ZINC38027875 & 61010427 & 41.09894 & 45.90255 & -6 \\
\hline ZINC00124224 & 2745819,6927290 & 40.981876 & 44.329945 & -6 \\
\hline ZINC00424668 & 4413737 & 40.02153 & 44.9367 & -6 \\
\hline ZINC06928577 & 3352935 & 41.162056 & 43.946198 & -6 \\
\hline ZINC83237584 & 53270535 & 39.666634 & 44.377777 & -6 \\
\hline ZINC83237585 & 53270535 & 39.66785 & 45.874893 & -6 \\
\hline ZINC49448722 & 49736347 & 41.08783 & 43.5883 & -6 \\
\hline ZINC13690466 & 51526155,17596495 & 41.15819 & 43.07473 & -6 \\
\hline ZINC01520295 & 44184931 & 40.944496 & 43.50765 & -6 \\
\hline ZINC12955905 & 53399081 & 40.94205 & 43.701004 & -6 \\
\hline ZINC12367735 & 2825623,23252694 & 40.937172 & 44.69783 & -6 \\
\hline ZINC69150448 & 53500054 & 40.95651 & 45.89384 & -6 \\
\hline ZINC71969314 & 60506873 & 41.164814 & 44.290302 & -6 \\
\hline ZINC67713883 & 56706833 & 40.939514 & 45.544697 & -6 \\
\hline ZINC65370696 & 50948958 & 40.11453 & 45.00081 & -6 \\
\hline ZINC00170975 & 627521 & 41.189217 & 43.039165 & -6 \\
\hline ZINC01562166 & $\begin{array}{l}\text { 71102,6431733,71103,5091306, } \\
50989982,24189320,6915994, \\
54610113\end{array}$ & 40.053066 & 44.73631 & -6 \\
\hline ZINC03311576 & 2427297 & 41.038162 & 45.04168 & -6 \\
\hline ZINC41873562 & 47143012 & 41.149605 & 45.666245 & -6 \\
\hline
\end{tabular}




\begin{tabular}{|c|c|c|c|c|}
\hline ZINC16769937 & 6416378,27326114 & 40.782562 & 44.73593 & -6 \\
\hline ZINC19845023 & 28400709 & 39.994255 & 45.170437 & -6 \\
\hline ZINC33349127 & $5581287,40593087,1801231$ & 40.78524 & 44.03981 & -6 \\
\hline ZINC00097185 & \begin{tabular}{|l}
$6924709,708491,5396303$ \\
\end{tabular} & 41.13484 & 44.143604 & -6 \\
\hline ZINC49483146 & 49745583 & 41.231506 & 44.692474 & -6 \\
\hline ZINC38010196 & 54896736 & 40.249744 & 43.54377 & -6 \\
\hline ZINC04987582 & 6499373 & 39.643196 & 42.2114 & -6 \\
\hline ZINC67640764 & 56738073 & 39.485855 & 43.28148 & -6 \\
\hline ZINC36960276 & 43166622 & 40.022804 & 45.722992 & -6 \\
\hline ZINC35022494 & 51676158,44782902 & 39.877224 & 43.592937 & -6 \\
\hline ZINC02507584 & 3328703 & 40.169342 & 45.10894 & -6 \\
\hline ZINC08894787 & 44168178,4982969 & 40.115757 & 44.186535 & -6 \\
\hline ZINC36973977 & 61137131,43170874 & 39.943607 & 44.558865 & -6 \\
\hline ZINC40496947 & 45808301 & 40.169956 & 43.39449 & -6 \\
\hline ZINC04259343 & 11886021 & 40.448376 & 44.182224 & -6 \\
\hline ZINC39254991 & 12617481 & 41.159245 & 43.016518 & -6 \\
\hline ZINC20450375 & 28954219 & 41.357265 & 45.274284 & -6 \\
\hline ZINC37435237 & 61136713,43355565 & 40.18778 & 45.41163 & -6 \\
\hline ZINC82313476 & 68557352,13927273 & 40.03646 & 45.28691 & -6 \\
\hline ZINC12340014 & 2805326,5713954 & 39.705437 & 44.73936 & -6 \\
\hline ZINC38027852 & 61010423 & 40.04716 & 45.832542 & -6 \\
\hline ZINC22159504 & 30064393,16780466 & 39.572872 & 44.745174 & -6 \\
\hline ZINC49452847 & 67453196,53212170 & 39.62512 & 43.77049 & -5.9 \\
\hline ZINC69453403 & 53525978 & 39.68626 & 43.325066 & -5.9 \\
\hline ZINC75896538 & 66939153 & 40.802868 & 45.36264 & -5.9 \\
\hline ZINC65321310 & 53206499 & 39.868652 & 43.33674 & -5.9 \\
\hline ZINC06786115 & 1390611,6868074 & 39.962482 & 43.955338 & -5.9 \\
\hline ZINC38027853 & 61010423 & 40.047813 & 43.667385 & -5.9 \\
\hline ZINC65528363 & 50983600 & 41.1153 & 45.672173 & -5.9 \\
\hline ZINC65528362 & 50983600 & 41.1153 & 45.679237 & -5.9 \\
\hline ZINC39647696 & 13436395 & 40.509575 & 44.82385 & -5.9 \\
\hline ZINC71405884 & 56861754 & 40.676373 & 45.307453 & -5.9 \\
\hline ZINC67788571 & 56715550 & 40.430893 & 44.375084 & -5.9 \\
\hline ZINC38006881 & 60999807 & 41.125492 & 43.972366 & -5.9 \\
\hline ZINC20075571 & 28600841 & 40.106247 & 44.623657 & -5.9 \\
\hline ZINC69451210 & 53565477 & 41.117626 & 44.32704 & -5.9 \\
\hline ZINC48345792 & 47054873 & 40.039413 & 43.610733 & -5.9 \\
\hline ZINC08549344 & 40768027,4775720 & 39.58043 & 43.78855 & -5.9 \\
\hline ZINC54752331 & 60527954 & 41.188526 & 43.684532 & -5.9 \\
\hline ZINC68589860 & 54592726 & 41.36356 & 43.996353 & -5.9 \\
\hline ZINC05256619 & 7590097 & 39.841362 & 44.17256 & -5.9 \\
\hline ZINC22159493 & \begin{tabular}{|l|l}
$57367593,30064379,16780466$ \\
\end{tabular} & 39.573227 & 44.743843 & -5.9 \\
\hline ZINC65386155 & 50956654 & 40.976177 & 45.287743 & -5.9 \\
\hline ZINC02147563 & 51383035,2743678 & 41.187645 & 44.56572 & -5.9 \\
\hline ZINC31973272 & 39196115 & 39.483753 & 44.982517 & -5.9 \\
\hline ZINC37435236 & 43355565 & 40.18778 & 45.210117 & -5.9 \\
\hline ZINC14180454 & 17460266 & 40.805805 & 45.309 & -5.9 \\
\hline ZINC71915984 & 56794177 & 41.044865 & 44.458443 & -5.9 \\
\hline ZINC65321311 & 53206499 & 39.868652 & 44.85305 & -5.9 \\
\hline ZINC39289832 & 16637919 & 40.29741 & 43.482807 & -5.9 \\
\hline ZINC32918521 & 44122305 & 41.101646 & 43.02125 & -5.9 \\
\hline ZINC20386074 & 28906396,21087403 & 40.306126 & 44.987274 & -5.9 \\
\hline ZINC40448176 & 51980191,51064028 & 41.179726 & 44.777 & -5.9 \\
\hline ZINC49549216 & 52322129,51054187 & 40.838707 & 44.621605 & -5.9 \\
\hline ZINC04243799 & \begin{tabular}{|l|l}
51709101,7147525 \\
\end{tabular} & 40.05874 & 45.20871 & -5.9 \\
\hline ZINC02392762 & 24723377 & 40.390343 & 45.081932 & -5.9 \\
\hline ZINC05701581 & 1481808,40556518 & 41.159798 & 42.81779 & -5.9 \\
\hline
\end{tabular}




\begin{tabular}{|c|c|c|c|c|}
\hline ZINC44979306 & 45834860 & 41.446228 & 44.61345 & -5.9 \\
\hline ZINC71922345 & 56807196 & 40.107506 & 43.848076 & -5.9 \\
\hline ZINC72148041 & 56893331 & 41.1153 & 44.581764 & -5.9 \\
\hline ZINC12997733 & 25658382,42932171 & 39.99476 & 43.30632 & -5.9 \\
\hline ZINC40496945 & 45808301 & 40.169956 & 43.39449 & -5.9 \\
\hline ZINC02582688 & 7022942,4714955 & 39.967125 & 44.71441 & -5.9 \\
\hline ZINC38490415 & 45505156 & 41.171654 & 43.20829 & -5.9 \\
\hline ZINC83662599 & 64282205 & 41.16061 & 45.121563 & -5.9 \\
\hline ZINC00121303 & 2744256,44160522 & 41.07915 & 43.867756 & -5.9 \\
\hline ZINC38022549 & 61006963 & 40.215782 & 44.75356 & -5.9 \\
\hline ZINC16682101 & $51564576,27276482,43835780$ & 40.178806 & 43.28148 & -5.9 \\
\hline ZINC04002446 & $2766702,3632531,51398372$ & 41.176468 & 44.32051 & -5.9 \\
\hline ZINC40496946 & 45808301 & 40.169956 & 43.533463 & -5.9 \\
\hline ZINC20295429 & 28823755 & 39.831528 & 44.452904 & -5.8 \\
\hline ZINC22065932 & $29986484,13450921,23722420$ & 40.050694 & 44.884808 & -5.8 \\
\hline ZINC06192230 & 1801231,9560533 & 40.858337 & 44.03884 & -5.8 \\
\hline ZINC19344763 & 46952439 & 41.18145 & 43.567368 & -5.8 \\
\hline ZINC32750812 & 51658836,39972513 & 40.795876 & 42.84822 & -5.8 \\
\hline ZINC40544125 & 45829240 & 40.187614 & 43.80071 & -5.8 \\
\hline ZINC26438989 & 14567875,33695724 & 39.92838 & 43.52967 & -5.8 \\
\hline ZINC69596722 & 53585582 & 41.04707 & 43.262047 & -5.8 \\
\hline ZINC37406457 & 43238164 & 39.83281 & 44.319977 & -5.8 \\
\hline ZINC02787833 & 4774793,51390843 & 40.461708 & 43.882957 & -5.8 \\
\hline ZINC75898453 & 59580006 & 41.122055 & 45.497414 & -5.8 \\
\hline ZINC84193630 & 65808988 & 39.742775 & 43.688213 & -5.8 \\
\hline ZINC53168595 & 62531332 & 39.515106 & 43.639545 & -5.8 \\
\hline ZINC41067095 & 61269589 & 41.157246 & 44.969765 & -5.8 \\
\hline ZINC48945357 & 47382764 & 41.024082 & 44.4607 & -5.8 \\
\hline ZINC13563162 & 1205769,2844823 & 39.841633 & 44.17018 & -5.8 \\
\hline ZINC05672488 & 40554140,6819828 & 40.783398 & 43.6007 & -5.8 \\
\hline ZINC20357855 & 28875648 & 40.868298 & 45.013863 & -5.8 \\
\hline ZINC06817047 & 22423975 & 40.318943 & 43.74303 & -5.8 \\
\hline ZINC65397066 & 50960814 & 41.143833 & 44.909218 & -5.8 \\
\hline ZINC35672445 & 51699407,46300352 & 41.180714 & 45.561344 & -5.8 \\
\hline ZINC00111857 & 4132299,715387 & 40.6767 & 45.58589 & -5.8 \\
\hline ZINC67678970 & 50967371 & 39.539062 & 45.317757 & -5.8 \\
\hline ZINC77435043 & 65022490 & 40.89947 & 43.490944 & -5.8 \\
\hline ZINC16904281 & 43826307,27408755 & 40.198605 & 44.75837 & -5.8 \\
\hline ZINC57551464 & 67079041 & 40.927387 & 44.49507 & -5.8 \\
\hline ZINC38022532 & 61006802 & 39.947292 & 45.740707 & -5.8 \\
\hline ZINC37742880 & 54932868 & 40.913036 & 43.046165 & -5.8 \\
\hline ZINC39233283 & 12461543 & 41.151154 & 43.309277 & -5.8 \\
\hline ZINC31706150 & 10402363 & 41.15372 & 44.78575 & -5.8 \\
\hline ZINC17744396 & 804979,27734999 & 40.002747 & 43.041077 & -5.8 \\
\hline ZINC07076421 & 8207041 & 40.988594 & 43.829742 & -5.8 \\
\hline ZINC43299420 & 52188976,50989315 & 41.123436 & 45.244293 & -5.8 \\
\hline ZINC37333378 & 43330825 & 40.187958 & 45.163467 & -5.7 \\
\hline ZINC33332433 & 1400053 & 39.96265 & 43.95559 & -5.7 \\
\hline ZINC09355187 & 41031505,16480585 & 40.778904 & 43.800858 & -5.7 \\
\hline ZINC31944805 & 39159365 & 39.7895 & 42.9216 & -5.7 \\
\hline ZINC12428409 & 16764728 & 41.006855 & 43.277237 & -5.7 \\
\hline ZINC78985963 & 60298200 & 41.046886 & 44.33993 & -5.7 \\
\hline ZINC78985969 & 60298200 & 41.046104 & 44.337063 & -5.7 \\
\hline ZINC38024497 & 61010621 & 39.582966 & 44.59236 & -5.7 \\
\hline ZINC01681741 & 51381225,242017 & 40.195843 & 45.049408 & -5.7 \\
\hline ZINC38027139 & 61010988 & 39.562626 & 43.988243 & -5.7 \\
\hline ZINC65386153 & 50956654 & 41.017063 & 45.679504 & -5.7 \\
\hline
\end{tabular}




\begin{tabular}{|c|c|c|c|c|}
\hline ZINC66347805 & 46864143 & 39.65324 & 44.044964 & -5.7 \\
\hline ZINC71915986 & 56794177 & 41.046635 & 44.375526 & -5.7 \\
\hline ZINC71915985 & 56794177 & 41.044106 & 43.162468 & -5.7 \\
\hline ZINC13638655 & $6882875,6741007,5400190$ & 39.64915 & 43.86403 & -5.7 \\
\hline ZINC49466307 & 53214343 & 41.23683 & 44.913654 & -5.7 \\
\hline ZINC34964300 & 43148571 & 41.132465 & 44.372112 & -5.7 \\
\hline ZINC34964302 & 43148572 & 41.083763 & 42.80437 & -5.7 \\
\hline ZINC36948345 & 43164217 & 40.646908 & 43.678417 & -5.7 \\
\hline ZINC48981008 & 52312491,49657853 & 40.277763 & 44.637726 & -5.7 \\
\hline ZINC20159418 & $5465085,6807856,5388282$ & 41.28111 & 44.86873 & -5.7 \\
\hline ZINC49568643 & 12423945 & 40.276535 & 43.32742 & -5.7 \\
\hline ZINC51171225 & 62278575 & 40.118073 & 43.769676 & -5.7 \\
\hline ZINC66738100 & 53605373 & 41.23419 & 43.204647 & -5.7 \\
\hline ZINC16904283 & 43826307,27408757 & 40.19844 & 44.579216 & -5.7 \\
\hline ZINC25819711 & 55097387 & 40.507683 & 43.694748 & -5.7 \\
\hline ZINC35856938 & 11580357 & 41.02012 & 43.67495 & -5.7 \\
\hline ZINC71893923 & 56786433 & 41.179035 & 43.60222 & -5.6 \\
\hline ZINC05934634 & 47305,40572489 & 41.036434 & 44.86187 & -5.6 \\
\hline ZINC49065657 & 52314705,47393651 & 40.964962 & 42.80121 & -5.6 \\
\hline ZINC65419830 & 50968082 & 41.0077 & 44.84777 & -5.6 \\
\hline ZINC68043329 & 56785079 & 39.474163 & 43.499355 & -5.6 \\
\hline ZINC63772460 & 51342087 & 40.379356 & 44.071068 & -5.6 \\
\hline ZINC33488505 & 3003060 & 40.863678 & 43.783585 & -5.6 \\
\hline ZINC14955016 & 11702273 & 39.552258 & 44.892277 & -5.6 \\
\hline ZINC08496737 & 4770942 & 40.53145 & 44.317627 & -5.6 \\
\hline ZINC38074200 & 61033692 & 40.886448 & 42.79874 & -5.6 \\
\hline ZINC32916963 & 44121552 & 41.13913 & 43.037758 & -5.6 \\
\hline ZINC29473836 & 10586950 & 39.698685 & 44.726112 & -5.6 \\
\hline ZINC00107064 & 3276160,713282 & 41.158646 & 44.807995 & -5.6 \\
\hline ZINC38074214 & 61033695 & 40.214478 & 42.918808 & -5.6 \\
\hline ZINC26422215 & 33678053 & 41.023678 & 44.065544 & -5.6 \\
\hline ZINC40448171 & 51980188,51064024 & 40.91616 & 43.733444 & -5.6 \\
\hline ZINC11887885 & $42253284,20992745,42253283$ & 41.07581 & 43.92187 & -5.6 \\
\hline ZINC11827889 & $42203912,22686647,42203911$ & 41.075798 & 43.92187 & -5.6 \\
\hline ZINC15987730 & $44167485,6403976,1390611$ & 39.962482 & 43.955338 & -5.6 \\
\hline ZINC66738102 & 53605373 & 41.23419 & 43.967102 & -5.6 \\
\hline ZINC38482729 & 10314099 & 40.339783 & 44.487198 & -5.6 \\
\hline ZINC36719715 & 51869040,45490086 & 40.103615 & 45.66921 & -5.5 \\
\hline ZINC69841359 & 53610027 & 40.934265 & 44.331966 & -5.5 \\
\hline ZINC14611333 & 53214336 & 40.015263 & 45.591244 & -5.5 \\
\hline ZINC65371888 & 50949886 & 40.886593 & 42.93403 & -5.5 \\
\hline ZINC32500438 & 39732837 & 40.138653 & 44.58255 & -5.5 \\
\hline ZINC29975053 & 37267235 & 41.44882 & 43.287395 & -5.5 \\
\hline ZINC29473831 & 10586950 & 39.699333 & 44.089455 & -5.5 \\
\hline ZINC61957844 & 52903562 & 41.141006 & 44.172066 & -5.5 \\
\hline ZINC70598291 & 64308292 & 40.65438 & 42.856403 & -5.5 \\
\hline ZINC13914412 & 51529478 & 40.77359 & 45.321857 & -5.5 \\
\hline ZINC40174389 & 43839963 & 41.054283 & 44.548748 & -5.5 \\
\hline ZINC32592844 & 39834244,39834243 & 40.95273 & 45.518166 & -5.5 \\
\hline ZINC37405519 & 43237432 & 40.03308 & 44.892128 & -5.4 \\
\hline ZINC15780124 & 3831768 & 39.8956 & 44.76217 & -5.4 \\
\hline ZINC04593424 & 5294947 & 40.169117 & 42.87093 & -5.4 \\
\hline ZINC37405717 & 43237536 & 41.099564 & 45.354034 & -5.4 \\
\hline ZINC83250450 & 53273844 & 40.526108 & 43.49025 & -5.4 \\
\hline ZINC32918392 & 44122243 & 39.9605 & 45.083817 & -5.4 \\
\hline ZINC67173925 & 46839887 & 39.79596 & 45.218567 & -5.4 \\
\hline ZINC62469070 & 66043411 & 41.088608 & 44.845097 & -5.4 \\
\hline
\end{tabular}




\begin{tabular}{|l|l|l|l|l|}
\hline ZINC06649926 & 7131178,40633240 & 41.148266 & 45.077484 & -5.4 \\
\hline ZINC34929199 & 51674824,42647357 & 40.009552 & 45.61472 & -5.4 \\
\hline ZINC38015436 & 61021235 & 39.569725 & 43.882797 & -5.4 \\
\hline ZINC62469152 & 66046044 & 41.088608 & 45.61875 & -5.4 \\
\hline ZINC71915983 & 56794177 & 41.045277 & 43.03801 & -5.4 \\
\hline ZINC38015587 & 61022229 & 40.41717 & 43.816204 & -5.4 \\
\hline ZINC72416954 & 66490447 & 39.414436 & 44.57848 & -5.3 \\
\hline ZINC01503031 & 5191259 & 41.200455 & 43.935905 & -5.3 \\
\hline ZINC38017995 & 54900489 & 40.990326 & 45.195087 & -5.3 \\
\hline ZINC49452978 & 53212257 & 39.79388 & 45.299866 & -5.3 \\
\hline ZINC83239743 & 53271721 & 40.225197 & 43.523396 & -5.3 \\
\hline ZINC20581644 & 29063100,12317280 & 40.29823 & 43.6454 & -5.3 \\
\hline ZINC37729203 & 60908158 & 40.991203 & 44.825207 & -5.3 \\
\hline ZINC00039816 & $16409899,3359873,676586$ & 39.738815 & 44.892586 & -5.3 \\
\hline ZINC02548189 & $2760621,5706557,7018296$ & 40.270203 & 46.0122 & -5.3 \\
\hline ZINC51172791 & 62276893 & 40.99081 & 44.824055 & -5.3 \\
\hline ZINC72415798 & 65208615 & 39.57422 & 45.009094 & -5.3 \\
\hline ZINC00390397 & 6950420,853341 & 39.741573 & 44.88993 & -5.3 \\
\hline ZINC67173990 & 46839951 & 39.66585 & 45.56922 & -5.2 \\
\hline ZINC39947660 & 51922075,47002544 & 41.175327 & 44.499554 & -5.2 \\
\hline ZINC38017367 & 54899996 & 39.79056 & 43.90236 & -5.2 \\
\hline ZINC70598351 & 64309551 & 40.96598 & 43.796627 & -5.2 \\
\hline ZINC08945152 & 17413704 & 40.59964 & 44.517574 & -5.2 \\
\hline ZINC37405520 & 43237432 & 40.03308 & 44.670055 & -5.1 \\
\hline ZINC50907388 & 62204574 & 40.956406 & 45.211643 & -5.1 \\
\hline ZINC09254117 & 40986952,16384372 & 39.911972 & 43.791855 & -5 \\
\hline ZINC04394469 & 2744149,44165613 & 41.102516 & 43.38402 & -4.9 \\
\hline ZINC01727104 & $5458303,422291,24192750$ & 41.134842 & 45.250965 & -4.8 \\
\hline ZINC65336727 & 50849256 & 39.616547 & 44.618027 & -4.7 \\
\hline
\end{tabular}

